\title{
The Gold Mineralization of the Baranyevskoe Au-Ag Epithermal Deposit in Central Kamchatka
}

\author{
Nadezhda Tolstykh ${ }^{1, *}$, Daria Bukhanova ${ }^{2}{ }^{\circ}$, Maria Shapovalova ${ }^{1}$, Andrey Borovikov ${ }^{1}$ and Maksim Podlipsky ${ }^{1}$ \\ 1 Sobolev Institute of Geology and Mineralogy of SB RAS, 630090 Novosibirsk, Russia; \\ shapovalovam@igm.nsc.ru (M.S.); borovikov@igm.nsc.ru (A.B.); maxim@igm.nsc.ru (M.P.) \\ 2 Institute of Volcanology and Seismology FEB RAS, 683006 Petropavlovsk-Kamchatsky, Russia; \\ dasha-snejinka@yandex.ru \\ * Correspondence: tolst@igm.nsc.ru
}

Citation: Tolstykh, N.; Bukhanova, D.; Shapovalova, M.; Borovikov, A.; Podlipsky, M. The Gold

Mineralization of the Baranyevskoe Au-Ag Epithermal Deposit in Central Kamchatka. Minerals 2021, 11, 1225. https://doi.org/10.3390/min11111225

\begin{abstract}
The Baranyevskoe Au-Ag epithermal deposit of low-sulfidation (LS) type is located on the Kamchatka Peninsula in the Neogene-Quaternary Central Kamchatka Volcanic Belt, where Aubearing quartz veins are usually accompanied by veinlet stockworks. Two economic associations are typical of the Baranyevskoe deposit. The first corresponds to gold-pyrite-quartz association with low-grade native gold (521-738\%00) intergrown with pyrite. Some accessory Au-Ag minerals within the early association were also identified: acanthite $\mathrm{AgS}_{2}$, hessite $\mathrm{AgTe}_{2}$, lenaite $\mathrm{Ag}(\mathrm{Fe}, \mathrm{Cu}) \mathrm{S}_{2}$, petzite $\mathrm{Ag}_{3} \mathrm{AuTe}_{2}$, utenbogardite $\mathrm{Ag}_{3} \mathrm{AuS}_{2}$ and unnamed $\mathrm{Ag}$-Sb-As sulfosalts. The former Au-Ag minerals were most likely formed in the temperature range of $320-330{ }^{\circ} \mathrm{C}$ based on the study of arsenopyrite thermometers and fluid inclusions. The second, a gold-sulfosalt-quartz association, includes highgrade native gold (883-941\%oo) in intergrowth with chalcopyrite. Cuprous phases (bornite, chalcocite, heerite, native copper, $\mathrm{Cu}-\mathrm{Zn}$ solid solutions), Bi-rich sulfosalts (aikinite $\mathrm{PbCuBiS}_{3}$, emplectite $\mathrm{CuBiS}_{2}$, witticenite $\mathrm{Cu}_{3} \mathrm{BiS}_{3}$ ), stannoidite $\mathrm{Cu}_{8} \mathrm{Fe}_{3} \mathrm{Sn}_{2} \mathrm{~S}_{12}$, mawsonite $\mathrm{Cu}_{6} \mathrm{Fe}_{2} \mathrm{SnS}_{8}$ ), Au-bearing galena, Te-free and Bi-rich tetrahedrite-tennantite represent this association. Fluid inclusions in gold-sulfosalt-quartz association are characterized by homogenization temperature ranging from 226 to $298{ }^{\circ} \mathrm{C}$, and salinity from 0.4 to 1.2 wt. \% $\mathrm{NaCl}$ eq.
\end{abstract}

Keywords: Baranyevskoe deposit; LS epithermal deposit; gold-pyrite-quartz association; goldsulfosalt-quartz association; Kamchatka Peninsula

\section{Introduction}

Epithermal Au-Ag deposits on the Kamchatka Peninsula are important sources of precious metals. These deposits are located in volcanic belts extending along the subduction zone [1,2], and occur in a geodynamic setting of island arc [3]. Among the aforementioned deposits stand out Asachinskoe, Ametistovoe, Ozernovskoe, Aginskoe, Rodnikovoe, Mutnovskoe, Maletoyvayam and others [4-11].

All these Au-Ag deposits, according to the classification of Corbett [12], belong to the low-sulfidation (LS) or quartz-adularia type, except for the recently described Maletoyvayam deposit, which belongs to the high-sulfidation (HS) or quartz-alunite type [13-15]. The Baranyevskoe Au-Ag epithermal deposit is of the LS type and estimated to be formed by near-neutral $\mathrm{pH}$ fluids [16,17]. It is located in the Kamchatka Peninsula, on the left bank of the Baranye stream (right tributary of the Balkhach River), approximately $60 \mathrm{~km}$ from the Milkovo village. The deposit was discovered in 1972 and, ever since, it has been explored by geologists of different mining companies (Koryakgeoldobycha CJSC, Kamchatka Gold OJSC, Kamchatka Gold Exploration LLC, etc.). The reserves of gold at the Baranyevskoe deposit are reported to be greater than 30 metric tons with an average grade of about $9 \mathrm{~g} / \mathrm{t}$ [18]. The composition of ores has been studied previously, and three main mineral associations were described: gold-quartz-carbonate-adularia, gold-ore stockworks and quartz combs or "brushes" [19]; the composition of gold was compared with other deposits 
of the Balkhach ore cluster at Central Kamchatka [20]. It was also identified three stages of ore formation corresponding to the (1) early gold-pyrite-quartz association in altered rocks located in the central part of the deposit, (2) gold-sulfosalt-quartz association, developed in the northeastern part of the deposit, and (3) gold-poor sulfide-quartz associated with late veins [18]. The Baranyevskoe deposit is considered to be the prototype model for the abovementioned deposits of Kamchatka [18]. Therefore, further studies on identification of typomorphic features of $\mathrm{Au}-\mathrm{Ag}$ mineralization in different zones, compositions of noble metals and associated unusual minerals is suggested, in order to contribute to a better understanding of the genetic processes and ore formation mechanism of one of the leading gold deposits in the Central Kamchatka mining region. This study aims to investigate in more detail the mineralogical and geochemical features of the two main profitable associations (gold-pyrite-quartz and gold-sulfosalt-quartz) belonging to different stages of ore formation within different zones of the Baranyevskoe deposit. These features are generally considered very important aspects of gold metallogeny in constraining genetical-ore processes [3].

\section{Materials and Methods}

The composition of the minerals was studied in the "Analytical Center for Multielement and Isotope Research" at the Sobolev Institute of Geology and Mineralogy of the Siberian Branch of the Russian Academy of Sciences (analyst M. Khlestov) both, in heavy concentrates of crushed samples mounted into polished sections and thin section of rocks. Microanalyzer Mira 3 (Tescan, Czech Republic) with an energy dispersive X-Ray (EDS Oxford X-Max 80) spectrometer was used for this purpose. Operating parameters were as follows: accelerating voltage $20 \mathrm{kV}$, probe current $1.6 \mathrm{nA}$, spectrum acquisition time on samples $20 \mathrm{~s}$. The resolution of the X-ray spectrometer was $126-127 \mathrm{eV}$ on the $\mathrm{Mn} \mathrm{K \alpha}$ line, and the probe size was $12 \mathrm{~nm}$. The beam diameter during the analyses varies within $2-5$ microns, depending on the average atomic number of the sample and the wavelength of the characteristic $X$-ray radiation. The detection limit for most elements is within the range $0.2-0.3 \mathrm{wt}$. \%. All analyses in the tables are given according to the detection limits of elements. The equivalence between measurements of EDS and WDS methods is given in [21].

Analysis of Au was performed at the Testing laboratory of AO SGS Vostok Limited, (Chita city) from a $30 \mathrm{~g}$ sample by means of assay analysis with NexIO 300D Inductively Coupled Plasma Optical Emission Spectroscopy (ICP-OES) ending; accreditation scope: $\mathrm{Au}-0.02 \mathrm{ppm}-20,000 \mathrm{ppm}$. All other elements were analyzed by a combined method including decomposition of the sample in a mixture of acids. After decomposition, the solution was analyzed by either Optima 8300 Inductively Coupled Plasma Atomic Emission Spectrometer (ICP-AES) or Inductively Coupled Plasma Mass Spectrometer (ICP-MS) (Perkin Elmer, Shetlon, Connecticut, USA).

Temperatures of homogenization and melting of solid phases in fluid inclusions were determined out using a Linkam THMSG-600 microthermal chamber (Linkam Scientific Instruments, Tadworth Surrey, United Kingdom, https://www.linkam.co.uk/, 11 October 2021 visited) with a measurement range of -196 to $+600^{\circ} \mathrm{C}$. The salinity of fluid inclusions and classification into a water-salt system were determined from cryometry data [22-25]. When assessing the pressure of ore formation according to the study of fluid inclusions, the computer program "AqSo_NaCl" was used [22].

\section{Geological Settings}

The Balkhach volcano-tectonic structure is located within the Neogene-Quaternary Central Kamchatka Volcanic Belt (CKVB), which extends for $800 \mathrm{~km}$ along the Main Kamchatka fault, striking north-east at its junction with the Sredinno-Kamchatka uplift composed by metamorphic rocks of different composition [26]. The formation and development of the circular Balkhach volcano-tectonic structure, with a diameter of $22 \mathrm{~km}$, is discussed in detail in [18]. The ore cluster of the same name within this structure combines 
four deposits: Baranyevskoe, Zolotoe, Kungurtsevskoe and Uglovoe. The Aginsk volcanotectonic structure, which includes the Aginskoe gold deposit, is located to the northwest of the Balkhach volcano-tectonic structure (Figure 1). The Baranyevskoe deposit belongs to the Baranyevskoe ore field and the Kungurtsevskoe and Zolotoe deposits to the Zolotoe ore field (Figure 2).
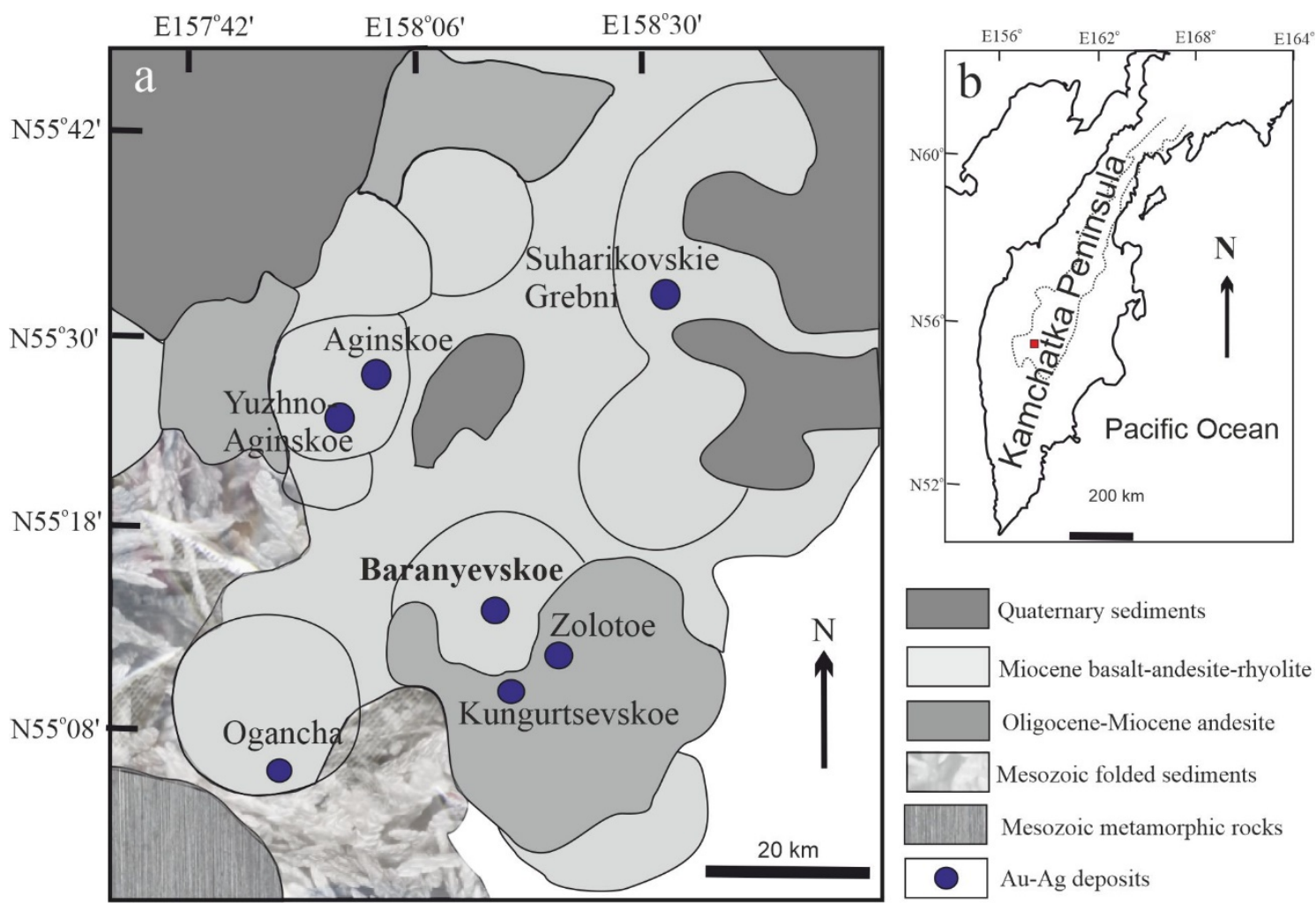

Figure 1. Location of the Au-Ag deposits on the geological map of the Central Kamchatka ore region modified after [18,26] (a) and their position on the Kamchatka Peninsula (red square) in the contours of the Central Kamchatka volcanic belt (b).

The stratigraphy of the Baranyevskoe ore field includes tuff of intermediate and mafic composition, basalt, trachyandesite, tuffaceous sandstone, tuffaceous siltstone (lower stratigraphical level); and effusive rocks and tuff of intermediate and mafic composition, andesite, basalt (upper structural level) (Figure 2). Ages of deposits of the Zolotoe ore field are: Kungurtsevskoe-21 Ma [20], Zolotoe-in the interval of 21.3-17.0 Ma, whereas the Baranyevskoe deposit, located in Late Miocene-Pliocene rocks, has an age interval of 3.9-2.4 Ma according to the K-Ar method [27].

The Baranyevskoe deposit consists of a system of vein-veinlet and stockwork (veinletdisseminated) ore-bearing structures in the zone of deep northeastern faulting (Figure 3). The Rusty ore zone is located along the central fault NE-SW and is accompanied by zones of abundant apophyses: the "Central", "Southern" and "Hanging", along with others branches from the axial fault in the hanging wall [18].

Quartz veins are accompanied by veinlet-disseminated stockworks, on top of which there is a 200-m interval of rocks with high profusion of weakly mineralized (less than $1 \mathrm{ppm} \mathrm{Au}$ ) veinlets of carbonate and zeolite-carbonate compositions. On the other hand, the stockwork includes the rich vein-disseminated gold mineralization with an Au concentration up to $20 \mathrm{ppm}$, accompanied by metasomatic associations: pyrite-hematite-magnetitesericite (alunite)-quartz in the central part of the stockwork and pyrite-sericite-illite-quartz at the periphery. Vuggy silica is common at deeper levels of stockworks [18]. The proportion of mineralized veinlets overlapping disseminated mineralization increases to the southwest. Quartz veins in the hanging wall are also accompanied by Au-bearing metasomatites comprising quartz, adularia, hydromica, carbonate and clay minerals. Thus, 
the main ore bodies (zones) of the Baranyevskoe deposit, up to $20 \mathrm{~m}$ thick, are composed of: (a) thick quartz veins, (b) disseminated-vein halos, and (c) sulfidized hydrothermalmetasomatic rocks. Consequently, the ore bodies exhibit a ribbon-like shape with a length up to $1500 \mathrm{~m}$, in which gold is unevenly distributed.
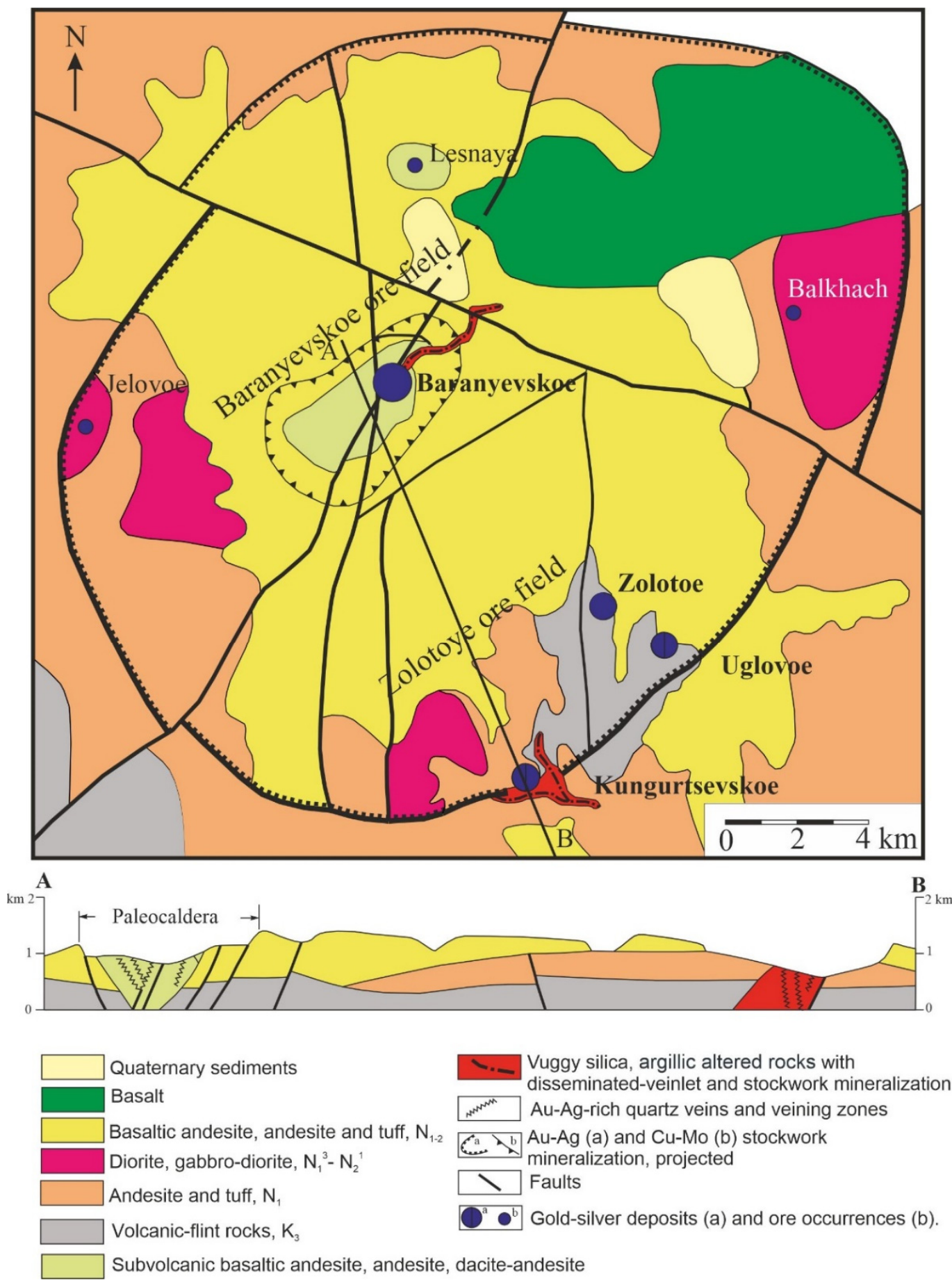

Figure 2. Geological-structural diagram of the Balkhach volcano-tectonic structure and respective A-B cross-section modified after $[18,26]$. 

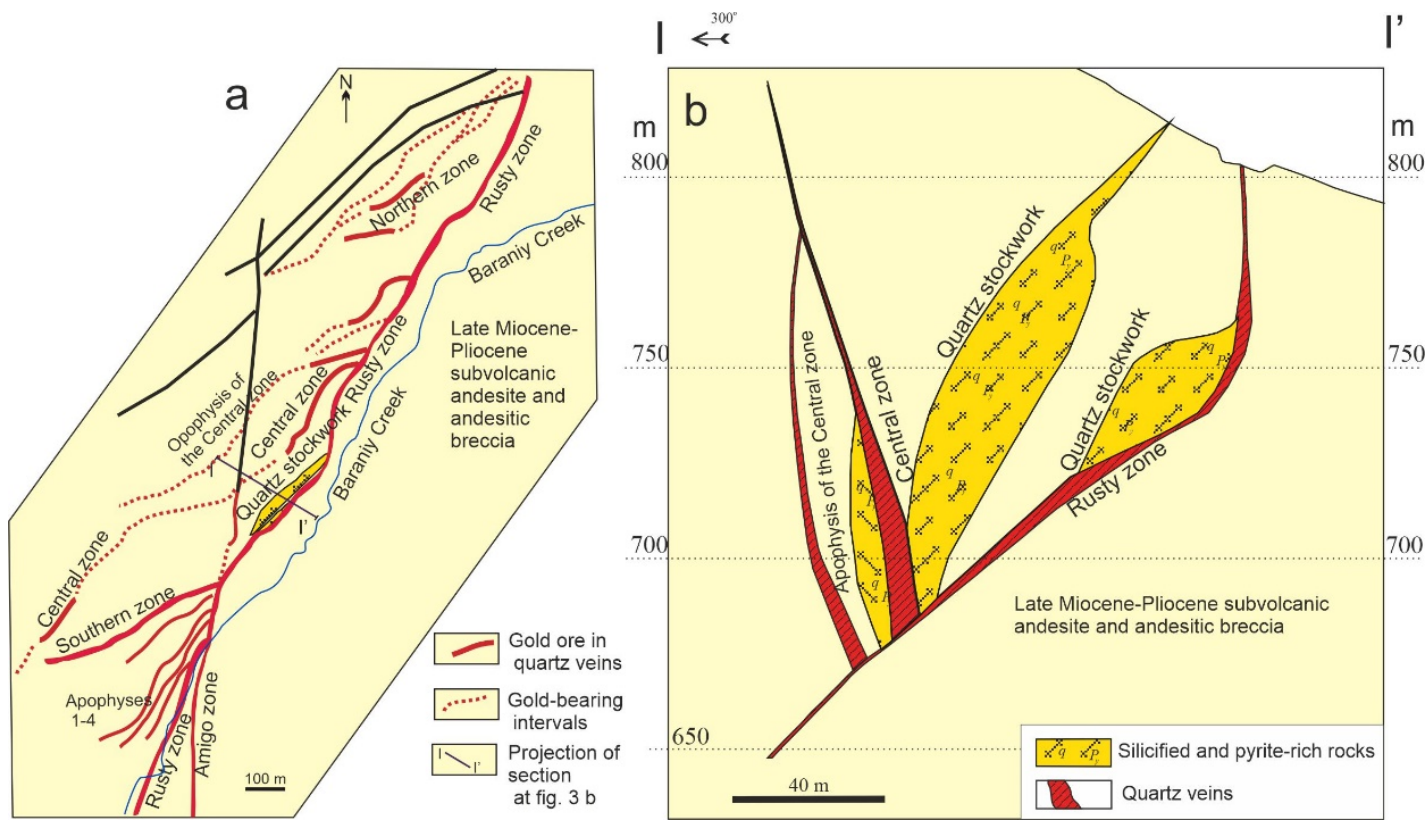

Figure 3. Geological scheme of the mineralization (a) and section I-I' (b) of the Baranyevskoe deposit, modified after [18]; vertical scale mean the absolute height above sea level $(\mathrm{m})$.

\section{Results}

\subsection{Samples}

The rock samples for this study were collected from an open pit in the Rusty, Central and Hanging zones, the underground tunnels of the Northern Zone and outcrops of the Southern Zone. Most of the samples gathered are characterized by the gold-pyrite-quartz association, which is the most widespread in the Central zone where metasomatic rocks are superimposed onto the gold-pyrite-quartz bearing veins. The most abundant mineral within this assemblage, pyrite, is sometimes replaced by hematite, whereas native gold may be found intergrown with pyrite. The role of the gold-sulfosalt-quartz association increases toward the northeastern direction of the deposit, and is more representative for the Northern zone. The gold-sulfosalt-quartz association is formed by samples of sericite-adularia-quartz composition with fragments of host-rock andesites, which are characterized, as a rule, by crustified-breccia textures (Figure 4a). Disseminated sulfides tend to form ribbon-like areas, due to the irregular flow of hydrothermal solutions. Additionally, native gold is found in paragenetic intergrowths with chalcopyrite (Figure 4b).
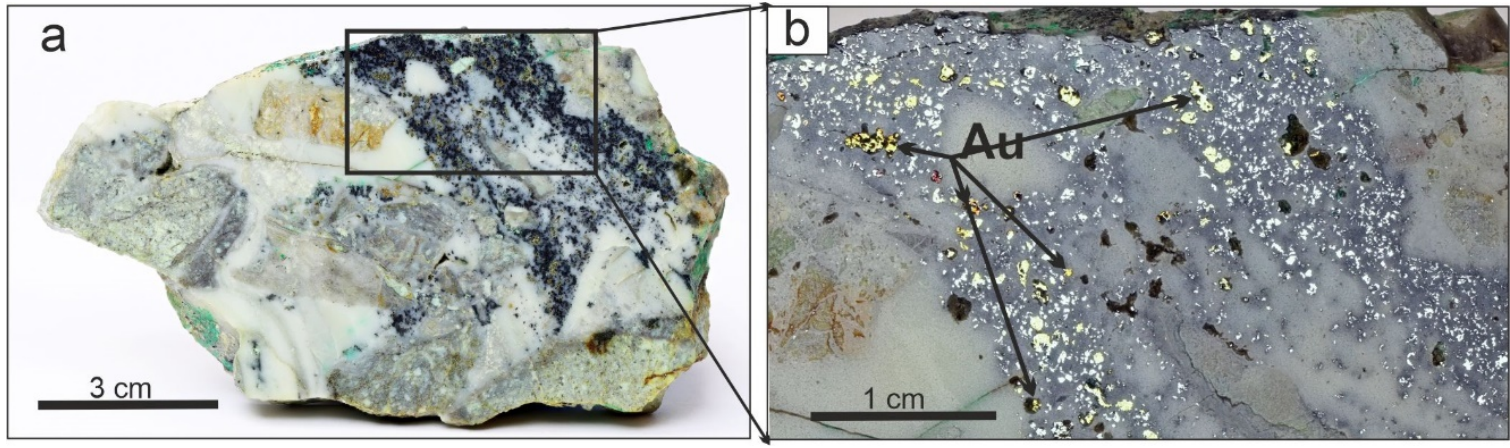

Figure 4. Sample of sericite-adularia-quartz composition with crustified-breccia texture containing disseminated sulfide and sulfosalts (a) and visible gold on an enlarged fragment of the sample (b) (sample Bar_2-1, Rusty zone). 


\subsection{The Basic Ore Minerals}

Pyrite is the most abundant sulfide at the Baranyevskoe deposit. Some analyses of pyrite (more than $30 \%$ ) contain As as a minor element. As-bearing pyrite forms intermittent growth zones with As-free pyrite grains (Figure $5 a, b)$. This oscillatory zoning observed in some pyrite grains and sulfosalts of epithermal deposits [28,29] is due to fluctuations in the characteristics of the hydrothermal fluid during the growth of minerals. The concentration of As in pyrite varies from grain to grain, reaching up to $7.4 \mathrm{wt}$. \% (Figure $5 \mathrm{c}$ ) while no other trace elements have so far been identified in the pyrite of the Baranyevskoe deposit (Table 1).
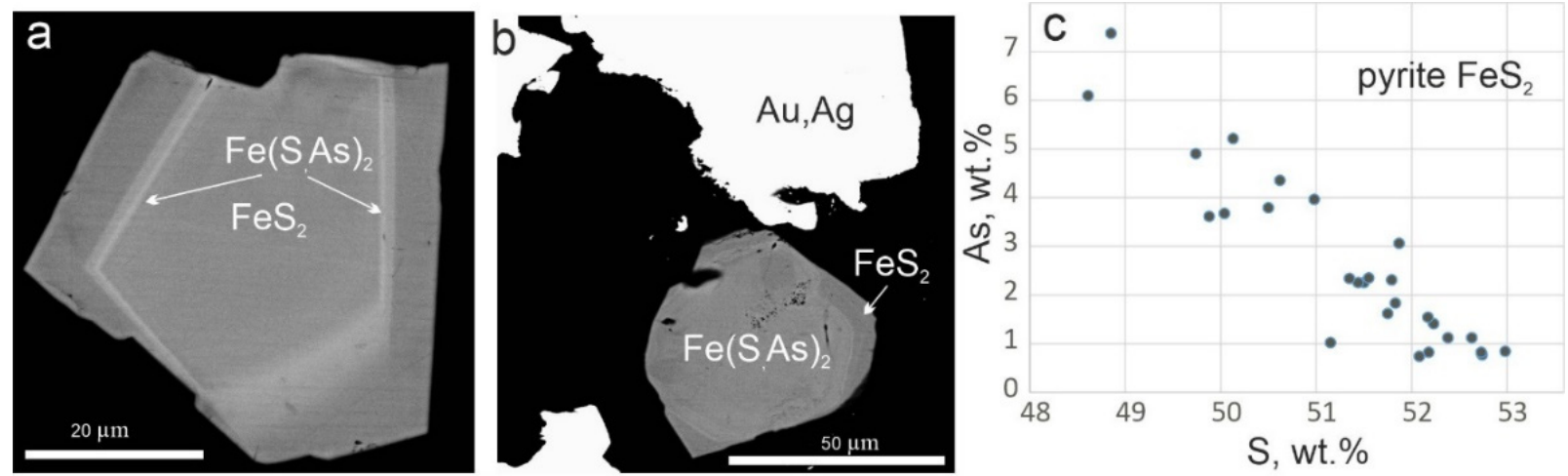

Figure 5. Back-scattering electron mode (BSE)-scanning electron microscope (SEM) images of As-containing pyrite grains $(\mathbf{a}, \mathbf{b})$ and the negative correlation $S$ versus As in pyrite of Baranyevskoe deposit (c).

Table 1. Composition of the main sulfides of the Baranyevskoe deposit, wt. \%.

\begin{tabular}{|c|c|c|c|c|c|c|c|c|c|}
\hline No. & Zone & Sample & $\mathrm{Fe}$ & $\mathrm{Cu}$ & $\mathbf{B i}$ & As & $S$ & Total & Formula \\
\hline 1 & Rusty & Bar-2-1 & 61.27 & & & & 38.67 & 99.94 & $\mathrm{Fe}_{0.95} \mathrm{~S}_{1.05}$ \\
\hline 2 & Rusty & Bar-2-1 & 60.73 & & & & 39.10 & 99.83 & $\mathrm{Fe}_{0.94} \mathrm{~S}_{1.06}$ \\
\hline 3 & Rusty & Bar-2-1 & 44.98 & & & 4.90 & 49.74 & 99.62 & $\mathrm{Fe}_{1.00}\left(\mathrm{~S}_{1.92} \mathrm{As}_{0.08}\right)_{2.00}$ \\
\hline 4 & Central & Bar-3-1 & 46.29 & & & 0.77 & 52.74 & 99.80 & $\mathrm{Fe}_{1.00}\left(\mathrm{~S}_{1.92} \mathrm{As}_{0.08}\right)_{2.00}$ \\
\hline 5 & Central & Bar-3-1 & 45.87 & & & 2.25 & 51.44 & 99.56 & $\mathrm{Fe}_{1.00}\left(\mathrm{~S}_{1.96} \mathrm{As}_{0.04}\right)_{2.00}$ \\
\hline 6 & Hanging & Bar-4 & 45.32 & & & 1.12 & 52.38 & 98.82 & $\mathrm{Fe}_{0.99}\left(\mathrm{~S}_{1.99} \mathrm{As}_{0.02}\right)_{2.01}$ \\
\hline 7 & Northern & Bar-5-1 & 46.19 & & & 0.82 & 52.73 & 99.74 & $\mathrm{Fe}_{1.00}\left(\mathrm{~S}_{1.99} \mathrm{As}_{0.01}\right)_{2.00}$ \\
\hline 8 & Northern & Bar-5-1 & 45.85 & & & 1.62 & 51.75 & 99.22 & $\mathrm{Fe}_{1.00}\left(\mathrm{~S}_{1.97} \mathrm{As}_{0.03}\right)_{2.00}$ \\
\hline 9 & Northern & Bar-5-1 & 45.72 & & & 2.31 & 51.79 & 99.82 & $\mathrm{Fe}_{1.00}\left(\mathrm{~S}_{1.97} \mathrm{As}_{0.04}\right)_{2.01}$ \\
\hline 10 & Northern & Bar-5-1 & 45.81 & & & 2.35 & 51.55 & 99.71 & $\mathrm{Fe}_{1.00}\left(\mathrm{~S}_{1.96} \mathrm{As}_{0.04}\right)_{2.00}$ \\
\hline 11 & Southern & Bar-6-5 & 45.36 & & & 3.96 & 50.98 & 100.30 & $\mathrm{Fe}_{0.99}\left(\mathrm{~S}_{1.94} \mathrm{As}_{0.06}\right)_{2.00}$ \\
\hline 12 & Southern & Bar-6-5 & 46.65 & & & 0.84 & 52.98 & 100.47 & $\mathrm{Fe}_{1.00}\left(\mathrm{~S}_{1.98} \mathrm{As}_{0.01}\right)_{1.99}$ \\
\hline 13 & Southern & Bar-6-5 & 45.15 & & & 4.35 & 50.62 & 100.12 & $\mathrm{Fe}_{0.99}\left(\mathrm{~S}_{1.94} \mathrm{As}_{0.07}\right)_{2.01}$ \\
\hline 14 & Southern & Bar-6-5 & 44.76 & & & 5.21 & 50.13 & 100.10 & $\mathrm{Fe}_{0.99}\left(\mathrm{~S}_{1.93} \mathrm{As}_{0.09}\right)_{2.02}$ \\
\hline 15 & Northern & Bar-5-1 & 44.61 & & & 7.37 & 48.95 & 100.93 & $\mathrm{Fe}_{0.99}\left(\mathrm{~S}_{1.89} \mathrm{As}_{0.12}\right)_{2.01}$ \\
\hline 16 & Northern & Bar-5-1 & 44.65 & & & 6.09 & 48.61 & 99.35 & $\mathrm{Fe}_{1.00}\left(\mathrm{~S}_{1.90} \mathrm{As}_{0.10}\right)_{2.00}$ \\
\hline 17 & Northern & Bar-5-2 & 29.93 & 34.39 & & & 34.87 & 99.19 & $\mathrm{Cu}_{1.00} \mathrm{Fe}_{0.99} \mathrm{~S}_{2.01}$ \\
\hline 18 & Northern & Bar-5-2 & 30.21 & 33.96 & & & 34.82 & 98.99 & $\mathrm{Cu}_{0.99} \mathrm{Fe}_{1.00} \mathrm{~S}_{2.01}$ \\
\hline 19 & Northern & Bar-5-2 & 30.09 & 33.67 & & & 34.78 & 98.54 & $\mathrm{Cu}_{0.98} \mathrm{Fe}_{1.00} \mathrm{~S}_{2.02}$ \\
\hline 20 & Northern & Bar-5-2 & 30.03 & 33.91 & & & 34.61 & 98.55 & $\mathrm{Cu}_{0.99} \mathrm{Fe}_{1.00} \mathrm{~S}_{2.01}$ \\
\hline 21 & Northern & Bar-5-2 & 29.7 & 33.91 & & & 34.5 & 98.11 & $\mathrm{Cu}_{1.00} \mathrm{Fe}_{0.99} \mathrm{~S}_{2.01}$ \\
\hline 22 & Rusty & Bar-2-1 & 30.28 & 34.38 & & & 34.58 & 99.24 & $\mathrm{Cu}_{1.00} \mathrm{Fe}_{1.00} \mathrm{~S}_{2.00}$ \\
\hline 23 & Rusty & Bar-2-1 & 30.95 & 34.5 & & & 34.52 & 99.97 & $\mathrm{Cu}_{1.00} \mathrm{Fe}_{1.02} \mathrm{~S}_{1.98}$ \\
\hline 24 & Rusty & Bar-2-1 & 11.67 & 61.02 & 2.01 & & 25.78 & 100.48 & $\mathrm{Cu}_{4.84} \mathrm{Fe}_{1.05} \mathrm{Bi}_{0.05} \mathrm{~S}_{4.06}$ \\
\hline 25 & Rusty & Bar-2-1 & 11.69 & 60.78 & 2.36 & & 25.81 & 100.64 & $\mathrm{Cu}_{4.83} \mathrm{Fe}_{1.06} \mathrm{Bi}_{0.06} \mathrm{~S}_{4.06}$ \\
\hline 26 & Rusty & Bar-2-1 & 11.98 & 55.46 & 7.35 & & 25.66 & 100.45 & $\mathrm{Cu}_{4.54} \mathrm{Fe}_{1.12} \mathrm{Bi}_{0.18} \mathrm{~S}_{4.16}$ \\
\hline 27 & Northern & Bar-5-2 & 11.15 & 63.05 & & & 25.62 & 99.82 & $\mathrm{Cu}_{4.98} \mathrm{Fe}_{1.00} \mathrm{~S}_{4.01}$ \\
\hline 28 & Northern & Bar-5-2 & 11.18 & 62.90 & & & 25.19 & 99.27 & $\mathrm{Cu}_{5.01} \mathrm{Fe}_{1.01} \mathrm{~S}_{3.98}$ \\
\hline
\end{tabular}


Two hundred grains of gold from different zones and areas of the Baranyevskoe deposit were analyzed. The complete $\mathrm{Au}-\mathrm{Ag}$ solid solution are subdivided, according to [30] into native gold - up to 30 atomic (at.) \% of Ag (fineness 1000-700\% $\%$ ), electrum (fineness 700-300\% 00 ), "küstelite" (mineral not approved by International Mineralogical Association) with fineness 300-100\%

The composition in non-oxidized samples varies from low-grade to high-grade gold with a gap in the isomorphic series in the range of Au 74-88 at. \%. Some gold grains from the Rusty and Central zones, most of the grains from the Hanging zone and half of the gold grains from the Northern zone consist of native gold and electrum with a range of fineness of 521-738\% (Figure 6, based on data in Table 2), which are characteristic of the gold-pyrite-quartz association of the early ore-forming stage. A significant part of gold grains from the Northern zone and some grains from the Hanging zone correspond to high-grade native gold (883-941\% gold-sulfosalt-quartz association. Electrum, "küstelite", and the high-grade native gold are representative in the Southern zone from sediments below of quartz veins and in oxidized ores. Oxidized ores of Southern and Central zones also contain the finest gold (920-280\% (Figure 7, Table 3). Thus, Au-Ag solid solutions of different compositions are involved in the formation ores in different-stage in almost all zones of the Baranyevskoe deposit.

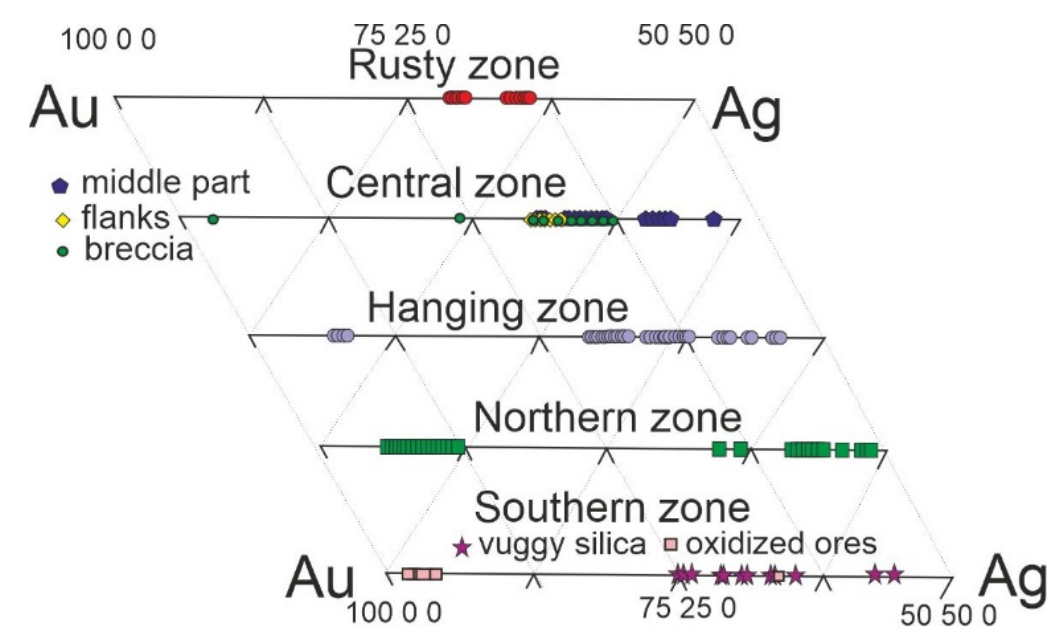

Figure 6. Variations in the composition of Au-Ag solid solutions from different ore zones of the Baranyevskoe deposit (data from Table 2).

Table 2. Composition of Au-Ag solid solutions from different zones of the Baranyevskoe deposit, wt. \%.

\begin{tabular}{cllccccccccc}
\hline No. & Zone & Sample & Au & Ag & Total & No. & Zone & Sample & Au & Ag & Total \\
\hline 1 & Rusty & Bar-2-1 & 77.87 & 23.43 & 101.30 & 81 & Hanging & Bar-4 & 79.41 & 20.29 & 99.70 \\
2 & Rusty & Bar-2-1 & 77.63 & 22.62 & 100.25 & 82 & Hanging & Bar-4 & 78.64 & 19.93 & 98.57 \\
3 & Rusty & Bar-2-1 & 76.66 & 22.23 & 98.89 & 83 & Hanging & Bar-4 & 79.81 & 20.15 & 99.96 \\
4 & Rusty & Bar-2-1 & 76.99 & 21.48 & 98.47 & 84 & Hanging & Bar-4 & 79.25 & 19.43 & 98.68 \\
5 & Rusty & Bar-2-1 & 78.03 & 20.37 & 98.40 & 85 & Hanging & Bar-4 & 79.27 & 18.77 & 98.04 \\
6 & Rusty & Bar-2-1 & 78.45 & 19.88 & 98.33 & 86 & Hanging & Bar-4 & 79.69 & 18.79 & 98.48 \\
7 & Rusty & Bar-2-1 & 78.53 & 19.89 & 98.42 & 87 & Hanging & Bar-4 & 82.23 & 19.15 & 101.38 \\
8 & Rusty & Bar-2-1 & 79.95 & 20.10 & 100.05 & 88 & Northern & Bar-5-1 & 65.76 & 33.14 & 98.90 \\
9 & Rusty & Bar-2-1 & 79.63 & 19.99 & 99.62 & 89 & Northern & Bar-5-1 & 66.77 & 32.66 & 99.43 \\
10 & Rusty & Bar-2-1 & 79.84 & 19.15 & 98.99 & 90 & Northern & Bar-5-1 & 67.10 & 32.40 & 99.50 \\
11 & Rusty & Bar-2-1 & 81.52 & 19.14 & 100.66 & 91 & Northern & Bar-5-1 & 67.13 & 31.06 & 98.19 \\
12 & Rusty & Bar-2-1 & 81.35 & 18.50 & 99.85 & 92 & Northern & Bar-5-1 & 69.54 & 31.54 & 101.08 \\
13 & Rusty & Bar-2-1 & 82.22 & 18.10 & 100.32 & 93 & Northern & Bar-5-1 & 70.12 & 30.55 & 100.67 \\
14 & Rusty & Bar-2-1 & 77.42 & 21.21 & 98.63 & 94 & Northern & Bar-5-1 & 70.11 & 30.33 & 100.44 \\
15 & Rusty & Bar-2-1 & 79.57 & 21.54 & 101.11 & 95 & Northern & Bar-5-1 & 70.26 & 30.29 & 100.55 \\
16 & Rusty & Bar-2-1 & 80.24 & 20.88 & 101.12 & 96 & Northern & Bar-5-1 & 70.10 & 29.82 & 99.92 \\
\hline
\end{tabular}


Table 2. Cont

\begin{tabular}{|c|c|c|c|c|c|c|c|c|c|c|c|}
\hline No. & Zone & Sample & $\mathbf{A u}$ & Ag & Total & No. & Zone & Sample & $\mathbf{A u}$ & Ag & Total \\
\hline 17 & Rusty & Bar-2-1 & 78.64 & 20.13 & 98.77 & 97 & Northern & Bar-5-1 & 70.20 & 29.80 & 100.00 \\
\hline 18 & Rusty & Bar-2-1 & 79.74 & 20.15 & 99.89 & 98 & Northern & Bar-5-1 & 69.71 & 29.32 & 99.03 \\
\hline 19 & Rusty & Bar-2-1 & 78.58 & 19.83 & 98.41 & 99 & Northern & Bar-5-1 & 69.13 & 29.01 & 98.14 \\
\hline 20 & Rusty & Bar-2-1 & 79.61 & 20.08 & 99.69 & 100 & Northern & Bar-5-1 & 70.82 & 29.12 & 99.94 \\
\hline 21 & Rusty & Bar-2-1 & 79.59 & 19.88 & 99.47 & 101 & Northern & Bar-5-1 & 70.4 & 28.79 & 99.19 \\
\hline 22 & Rusty & Bar-2-1 & 80.58 & 19.84 & 100.42 & 102 & Northern & Bar-5-1 & 71.33 & 28.95 & 100.28 \\
\hline 23 & Rusty & Bar-2-1 & 81.56 & 20.00 & 101.56 & 103 & Northern & Bar-5-1 & 71.8 & 29.10 & 100.90 \\
\hline 24 & Rusty & Bar-2-1 & 81.78 & 20.00 & 101.78 & 104 & Northern & Bar-5-1 & 71.52 & 28.55 & 100.07 \\
\hline 25 & Rusty & Bar-2-1 & 79.85 & 19.16 & 99.01 & 105 & Northern & Bar-5-1 & 71.56 & 28.55 & 100.11 \\
\hline 26 & Rusty & Bar-2-1 & 79.25 & 18.99 & 98.24 & 106 & Northern & Bar-5-1 & 72.46 & 28.51 & 100.97 \\
\hline 27 & Rusty & Bar-2-1 & 80.01 & 19.09 & 99.10 & 107 & Northern & Bar-5-1 & 71.82 & 28.00 & 99.82 \\
\hline 28 & Central & Bar-3 & 67.14 & 33.05 & 100.19 & 108 & Northern & Bar-5-1 & 70.57 & 27.46 & 98.03 \\
\hline 29 & Central & Bar-3 & 75.24 & 24.88 & 100.12 & 109 & Northern & Bar-5-1 & 71.54 & 27.48 & 99.02 \\
\hline 30 & Central & Bar-3 & 75.49 & 23.83 & 99.32 & 110 & Northern & Bar-5-1 & 75.77 & 24.03 & 99.80 \\
\hline 31 & Central & Bar-3 & 76.13 & 23.91 & 100.04 & 111 & Northern & Bar-5-1 & 77.16 & 22.74 & 99.90 \\
\hline 32 & Central & Bar-3 & 75.65 & 23.41 & 99.06 & 112 & Northern & Bar-5-2 & 70.47 & 30.21 & 100.68 \\
\hline 33 & Central & Bar-3 & 76.60 & 23.23 & 99.83 & 113 & Northern & Bar-5-2 & 70.62 & 29.64 & 100.26 \\
\hline 34 & Central & Bar-3 & 76.47 & 23.16 & 99.63 & 114 & Northern & Bar-5-2 & 70.09 & 29.12 & 99.21 \\
\hline 35 & Central & Bar-3 & 76.49 & 22.94 & 99.43 & 115 & Northern & Bar-5-2 & 70.18 & 28.61 & 98.79 \\
\hline 36 & Central & Bar-3 & 77.64 & 22.59 & 100.23 & 116 & Northern & Bar-5-2 & 70.59 & 28.60 & 99.19 \\
\hline 37 & Central & Bar-3 & 75.90 & 21.94 & 97.84 & 117 & Northern & Bar-5-2 & 71.08 & 28.49 & 99.57 \\
\hline 38 & Central & Bar-3 & 78.18 & 22.48 & 100.66 & 118 & Northern & Bar-5-2 & 71.90 & 28.35 & 100.25 \\
\hline 39 & Central & Bar-3 & 77.73 & 22.35 & 100.08 & 119 & Northern & Bar-5-2 & 71.30 & 28.07 & 99.37 \\
\hline 40 & Central & Bar-3 & 75.19 & 21.03 & 96.22 & 120 & Northern & Bar-5-2 & 73.78 & 28.44 & 102.22 \\
\hline 41 & Central & Bar-3-1 & 68.91 & 29.25 & 98.16 & 121 & Northern & Bar-5-2 & 92.61 & 7.21 & 99.82 \\
\hline 42 & Central & Bar-3-1 & 71.09 & 29.74 & 100.83 & 122 & Northern & Bar-5-2 & 93.04 & 6.76 & 99.80 \\
\hline 43 & Central & Bar-3-1 & 70.89 & 28.64 & 99.53 & 123 & Northern & Bar-5-2 & 93.67 & 6.79 & 100.46 \\
\hline 44 & Central & Bar-3-1 & 70.96 & 28.55 & 99.51 & 124 & Northern & Bar-5-2 & 93.76 & 6.40 & 100.16 \\
\hline 45 & Central & Bar-3-1 & 70.79 & 28.19 & 98.98 & 125 & Northern & Bar-5-2 & 93.80 & 6.25 & 100.05 \\
\hline 46 & Central & Bar-3-1 & 71.66 & 28.33 & 99.99 & 126 & Northern & Bar-5-2 & 93.15 & 6.17 & 99.32 \\
\hline 47 & Central & Bar-3-1 & 71.88 & 27.37 & 99.25 & 127 & Northern & Bar-5-2 & 91.42 & 6.05 & 97.47 \\
\hline 48 & Central & Bar-3-1 & 75.84 & 24.11 & 99.95 & 128 & Northern & Bar-5-2 & 90.41 & 5.96 & 96.37 \\
\hline 49 & Central & Bar-3-1 & 74.11 & 23.23 & 97.34 & 129 & Northern & Bar-5-2 & 93.56 & 6.03 & 99.59 \\
\hline 50 & Central & Bar-3-1 & 77.10 & 23.29 & 100.39 & 130 & Northern & Bar-5-2 & 93.70 & 5.87 & 99.57 \\
\hline 51 & Central & Bar-3-1 & 77.95 & 23.24 & 101.19 & 131 & Northern & Bar-5-2 & 93.47 & 5.77 & 99.24 \\
\hline 52 & Central & Bar-3-1 & 75.99 & 21.79 & 97.78 & 132 & Northern & Bar-5-2 & 95.25 & 5.80 & 101.05 \\
\hline 53 & Central & Bar-3-1 & 77.82 & 22.28 & 100.1 & 133 & Northern & Bar-5-2 & 93.93 & 5.64 & 99.57 \\
\hline 54 & Central & Bar-3-1 & 78.69 & 21.93 & 100.62 & 134 & Northern & Bar-5-2 & 92.81 & 5.50 & 98.31 \\
\hline 55 & Central & Bar-3-1 & 79.07 & 20.44 & 99.51 & 135 & Northern & Bar-5-2 & 95.73 & 5.50 & 101.23 \\
\hline 56 & Central & Bar-3-1 & 79.67 & 19.90 & 99.57 & 136 & Northern & Bar-5-2 & 92.60 & 5.23 & 97.83 \\
\hline 57 & Hanging & Bar-4 & 69.85 & 29.54 & 99.39 & 137 & Northern & Bar-5-2 & 92.83 & 5.23 & 98.06 \\
\hline 58 & Hanging & Bar-4 & 70.27 & 27.52 & 97.79 & 138 & Northern & Bar-5-2 & 93.59 & 4.78 & 98.37 \\
\hline 59 & Hanging & Bar-4 & 72.21 & 27.61 & 99.82 & 139 & Northern & Bar-5-2 & 94.28 & 4.62 & 98.90 \\
\hline 60 & Hanging & Bar-4 & 75.04 & 25.34 & 100.38 & 140 & Northern & Bar-5-2 & 93.88 & 4.54 & 98.42 \\
\hline 61 & Hanging & Bar-4 & 75.49 & 24.79 & 100.28 & 141 & Northern & Bar-5-2 & 96.58 & 4.13 & 100.71 \\
\hline 62 & Hanging & Bar-4 & 82.03 & 18.76 & 100.79 & 142 & Northern & Bar-5-2 & 95.20 & 4.00 & 99.20 \\
\hline 63 & Hanging & Bar-4 & 93.65 & 4.65 & 98.30 & 143 & Northern & Bar-5-2 & 94.98 & 3.87 & 98.85 \\
\hline 64 & Hanging & Bar-4 & 93.65 & 4.59 & 98.24 & 144 & Northern & Bar-5-2 & 96.27 & 3.86 & 100.13 \\
\hline 65 & Hanging & Bar-4 & 92.78 & 4.51 & 97.29 & 145 & Northern & Bar-5-2 & 95.01 & 3.71 & 98.72 \\
\hline 66 & Hanging & Bar-4 & 93.64 & 4.41 & 98.05 & 146 & Northern & Bar-5-2 & 96.79 & 3.48 & 100.27 \\
\hline 67 & Hanging & Bar-4 & 96.34 & 4.26 & 100.60 & 147 & Northern & Bar-5-2 & 95.15 & 3.29 & 98.44 \\
\hline 68 & Hanging & Bar-4 & 68.19 & 32.11 & 100.30 & 148 & Southern & Bar-6-1 & 68.41 & 31.16 & 99.57 \\
\hline 69 & Hanging & Bar-4 & 74.08 & 25.45 & 99.53 & 149 & Southern & Bar-6-1 & 70.36 & 29.79 & 100.15 \\
\hline 70 & Hanging & Bar-4 & 73.45 & 24.78 & 98.23 & 150 & Southern & Bar-6-2 & 78.47 & 16.21 & 94.68 \\
\hline 71 & Hanging & Bar-4 & 76.43 & 23.51 & 99.94 & 151 & Southern & Bar-6-2 & 80.76 & 16.66 & 97.42 \\
\hline 72 & Hanging & Bar-4 & 78.34 & 22.27 & 100.61 & 152 & Southern & Bar-6-2 & 79.68 & 15.50 & 95.18 \\
\hline 73 & Hanging & Bar-4 & 77.32 & 21.95 & 99.27 & 153 & Southern & Bar-6-2 & 77.01 & 24.30 & 101.31 \\
\hline 74 & Hanging & Bar-4 & 77.70 & 22.01 & 99.71 & 154 & Southern & Bar-6-2 & 76.10 & 23.66 & 99.76 \\
\hline
\end{tabular}


Table 2. Cont.

\begin{tabular}{cccccccccccc}
\hline No. & Zone & Sample & Au & Ag & Total & No. & Zone & Sample & Au & Ag & Total \\
\hline 75 & Hanging & Bar-4 & 77.51 & 20.75 & 98.26 & 155 & Southern & Bar-6-2 & 76.06 & 21.98 & 98.04 \\
76 & Hanging & Bar-4 & 77.65 & 20.74 & 98.39 & 156 & Southern & Bar-6-2 & 77.42 & 20.20 & 97.62 \\
77 & Hanging & Bar-4 & 78.68 & 20.99 & 99.67 & 157 & Southern & Bar-6-2 & 76.28 & 19.54 & 95.82 \\
78 & Hanging & Bar-4 & 79.77 & 21.00 & 100.77 & 158 & Southern & Bar-6-2 & 78.05 & 20.10 & 98.15 \\
79 & Hanging & Bar-4 & 78.83 & 20.63 & 99.46 & 159 & Southern & Bar-6-2 & 79.66 & 20.26 & 99.92 \\
80 & Hanging & Bar-4 & 77.99 & 20.12 & 98.11 & 160 & Southern & Bar-6-2 & 80.14 & 20.75 & 100.89 \\
\hline
\end{tabular}

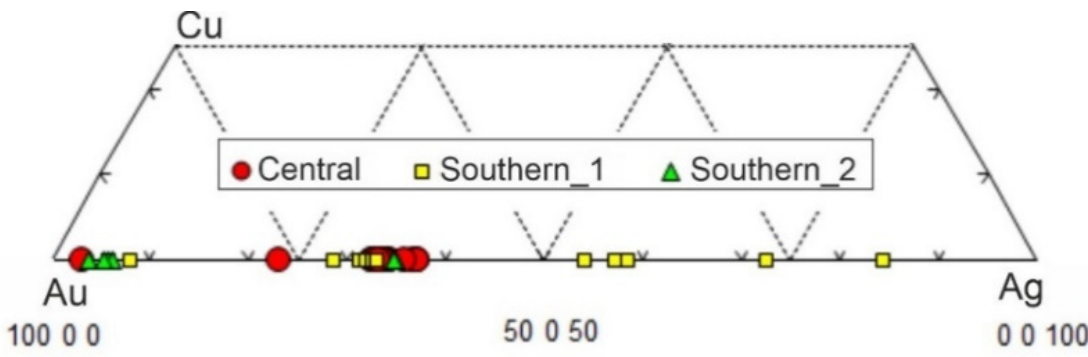

Figure 7. Variations in the composition of Au-Ag solid solutions from argillaceous-ocher sediments under mineralized outcrops of the Central and Southern zones. Southern _1 (samples BAR-6-2)— from sediments below quartz veins; Southern_2 (samples BAR-6-4)—under outcrops of oxidized ores (data from Table 3).

Table 3. Composition of Au-Ag solid solutions from argillaceous-ocher rocks underneath outcrops, wt. \%.

\begin{tabular}{|c|c|c|c|c|c|c|c|c|c|c|c|}
\hline No. & Zone & Sample & $\mathrm{Au}$ & Ag & Total & No. & Zone & Sample & $\mathrm{Au}$ & Ag & Total \\
\hline 1 & Central & Bar-3-1a & 97.04 & 1.73 & 98.77 & 20 & Central & Bar-3-1a & 76.86 & 21.04 & 97.90 \\
\hline 2 & Central & Bar-3-1a & 78.01 & 21.78 & 99.79 & 21 & Southern & Bar-6-2 & 80.77 & 17.74 & 98.51 \\
\hline 3 & Central & Bar-3-1a & 75.48 & 24.02 & 99.50 & 22 & Southern & Bar-6-2 & 60.76 & 39.20 & 99.96 \\
\hline 4 & Central & Bar-3-1a & 75.04 & 24.26 & 99.30 & 23 & Southern & Bar-6-2 & 57.28 & 41.95 & 99.23 \\
\hline 5 & Central & Bar-3-1a & 77.94 & 22.08 & 100.02 & 24 & Southern & Bar-6-2 & 39.98 & 57.67 & 97.65 \\
\hline 6 & Central & Bar-3-1a & 77.92 & 21.30 & 99.22 & 25 & Southern & Bar-6-2 & 25.48 & 75.53 & 101.01 \\
\hline 7 & Central & Bar-3-1a & 76.29 & 20.81 & 97.10 & 26 & Southern & Bar-6-2 & 80.08 & 20.02 & 100.100 \\
\hline 8 & Central & Bar-3-1a & 75.84 & 20.77 & 96.61 & 27 & Southern & Bar-6-2 & 77.41 & 19.80 & 97.21 \\
\hline 9 & Central & Bar-3-1a & 74.57 & 24.17 & 98.74 & 28 & Southern & Bar-6-2 & 56.68 & 43.77 & 100.45 \\
\hline 10 & Central & Bar-3-1a & 78.04 & 20.63 & 98.67 & 29 & Southern & Bar-6-2 & 77.98 & 20.63 & 98.61 \\
\hline 11 & Central & Bar-3-1a & 77.08 & 20.48 & 97.56 & 30 & Southern & Bar-6-2 & 96.73 & 3.30 & 100.03 \\
\hline 12 & Central & Bar-3-1a & 75.64 & 21.54 & 97.18 & 31 & Southern & Bar-6-2 & 94.18 & 4.47 & 98.65 \\
\hline 13 & Central & Bar-3-1a & 75.21 & 21.51 & 96.72 & 32 & Southern & Bar-6-2 & 78.60 & 21.15 & 99.75 \\
\hline 14 & Central & Bar-3-1a & 76.77 & 21.48 & 98.25 & 33 & Southern & Bar-6-4 & 96.63 & 3.28 & 99.91 \\
\hline 15 & Central & Bar-3-1a & 76.86 & 21.49 & 98.35 & 34 & Southern & Bar-6-4 & 74.84 & 22.12 & 96.96 \\
\hline 16 & Central & Bar-3-1a & 73.33 & 22.53 & 95.86 & 35 & Southern & Bar-6-4 & 96.69 & 2.11 & 98.80 \\
\hline 17 & Central & Bar-3-1a & 81.46 & 13.44 & 94.90 & 36 & Southern & Bar-6-4 & 96.43 & 3.55 & 99.98 \\
\hline 18 & Central & Bar-3-1a & 79.14 & 21.06 & 100.20 & 37 & Southern & Bar-6-4 & 95.53 & 2.92 & 98.45 \\
\hline 19 & Central & Bar-3-1a & 77.35 & 21.39 & 98.74 & 38 & Southern & Bar-6-4 & 76.62 & 22.43 & 99.05 \\
\hline
\end{tabular}

Note. Samples Bar-3-1a—from oxidized breccia; Bar-6-2—ochery-argillaceous highly altered rock under quartz veins, and Bar-6-4—under the outcrops of oxidized ores.

\subsection{Mineral Associations of Ore-Forming Stages}

\subsubsection{Gold-Pyrite-Quartz Association}

The gold-pyrite-quartz mineralization is more widespread in the central part of the deposit (Rusty zone), where metasomatites are overprinted on veinlets of gold-pyrite-quartz veins. The low-grade gold (electrum), as a rule, was found as individual grains in concentrates of crushed sample. Such gold grains are inclusions in pyrite, and associated with acanthite $\mathrm{Ag}_{2} \mathrm{~S}$, petzite $\mathrm{Ag}_{3} \mathrm{AuTe}_{2}$ and uytenbogardtite $\mathrm{Ag}_{3} \mathrm{AuS}_{2}$ (Figure 8a). In the Central zone, this mineral assemblage is characterized by inclusions of pyrite crystals in gold (Figure $8 \mathrm{~d}, \mathrm{e}$ ). Gold is associated with hessite $\mathrm{Ag}_{2} \mathrm{Te}$ and galena $\mathrm{PbS}$ (Figure $8 \mathrm{~b}$ ). The gold-pyrite-quartz mineralization in the Southern zone is featured by the development of unusual composition in acanthite, with traces of $\mathrm{Sb}$, As and Se, and sometimes $\mathrm{Au}$ : $(\mathrm{Ag}, \mathrm{Au})_{2}(\mathrm{~S}, \mathrm{Sb}, \mathrm{As}, \mathrm{Se})$, 
which is found as individual grains in ocher-clay samples (Figure $8 \mathrm{~h}$ ). The silver sulfosalts $\mathrm{Ag}_{10}(\mathrm{Sb}, \mathrm{As}) \mathrm{S}_{5}$ and $\mathrm{Ag}_{17}(\mathrm{Sb}, \mathrm{As})_{2}(\mathrm{~S}, \mathrm{Se})_{10}$ composition are found both in the Southern and Central zones in the gold-pyrite-quartz mineralization (Table 4). The replacement of acanthite by lenaite $\mathrm{Ag}(\mathrm{Fe}, \mathrm{Cu}) \mathrm{S}_{2}$ occurs (Figure $\left.8 \mathrm{i}\right)$. Sulfides in intergrowth with gold are sometimes replaced by Fe-oxide (Figure 8c).
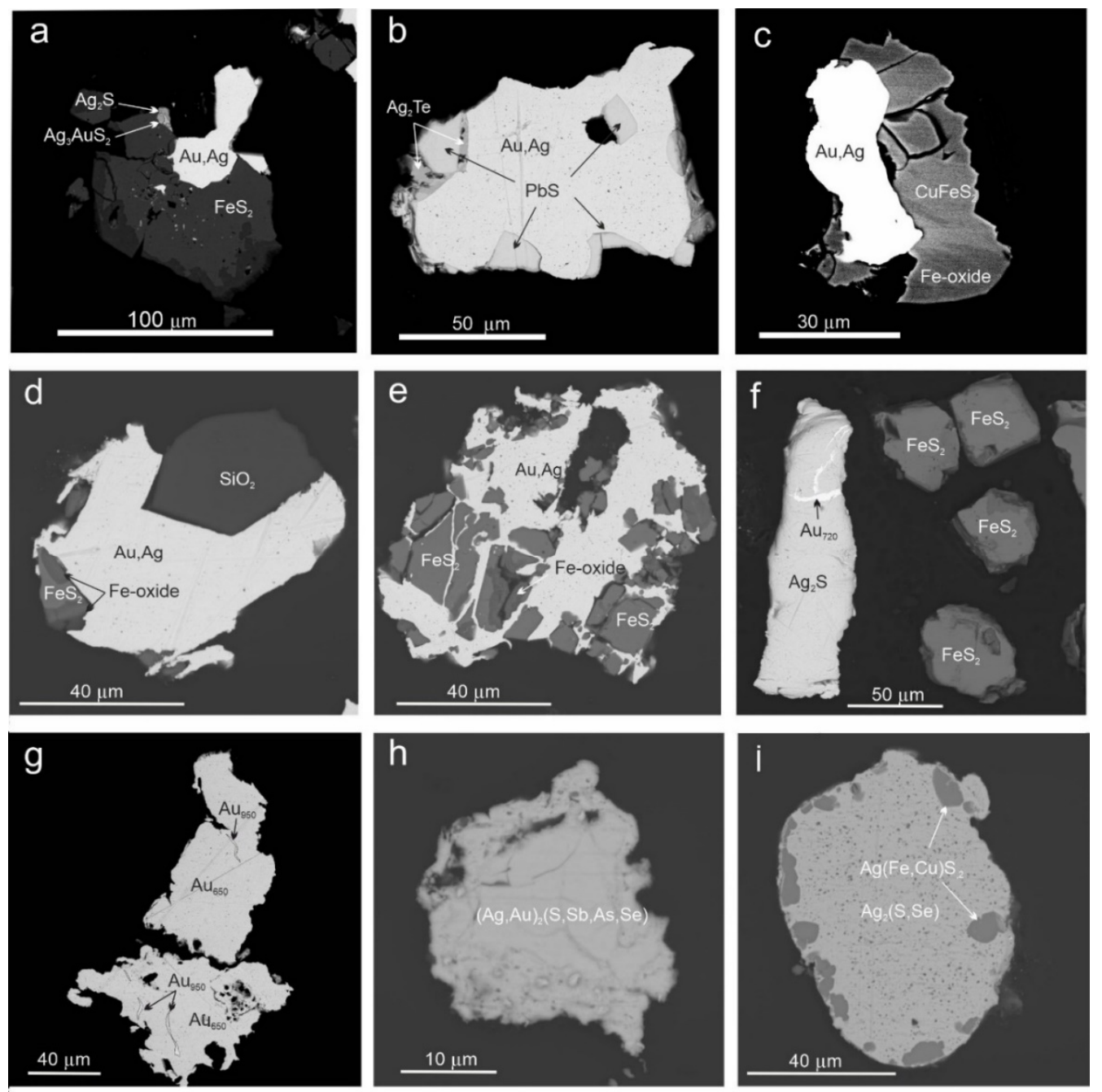

Figure 8. Microparagenesis of the gold-pyrite-quartz association: (a)-low-grade gold intergrown with pyrite FeS ${ }_{2}$ in assemblage with akantite $\mathrm{Ag}_{2} \mathrm{~S}$ and uytenbogardite $\mathrm{Ag}_{3} \mathrm{AuS}_{2}$, Rusty zone; (b)-low-grade gold in intergrowth with galena $\mathrm{PbS}$, hessite $\mathrm{Ag}_{2} \mathrm{Te}$ and petzite $\mathrm{Ag}_{3} \mathrm{AuTe}_{2}$, Rusty zone; (c-e)-low-grade gold in intergrowth with chalcopyrite and pyrite replaced by Fe-oxide, Central zone; (f) —-hessite with vein-like inclusions of low-grade gold; (g) —low-grade gold with vein-like inclusions of high-grade gold, South Zone; (h,i)—grains of Sb,As,Te-bearing akantite (Ag,Sb,As,Se) ${ }_{2} \mathrm{~S}$ sometimes replaced by lenaite $\mathrm{Ag}(\mathrm{Fe}, \mathrm{Cu}) \mathrm{S}_{2}$, the poorly compacted argillaceous-ocher rocks underneath the ore bodies, Southern zone.

\subsubsection{Gold-Sulfosalt-Quartz Association}

Native gold, belonging to this association, is intergrown with chalcopyrite, which is by far the most common mineral. Sample Bar-5-2 collected from underground mines of the Northern Zone is a typical example of a gold-sulfosalt-quartz association (Figure 9). The main mineral phase associated with gold is emplectite $\mathrm{CuBiS}_{2}$ (up to $50 \mu \mathrm{m}$ ), which occurs intergrown with gold or included in chalcopyrite in their joint paragenesis (Figure 9b,c,e-g,). Native gold within this mineral assemblage is always high-grade with a concentration of Ag no greater than 12 at. $\%$. 
Table 4. Composition of Au-Ag minerals in the gold-pyrite-quartz association from various zones of the Baranyevskoe deposit, wt. \%.

\begin{tabular}{|c|c|c|c|c|c|c|c|c|c|c|c|c|c|c|}
\hline No & Zone & $\mathrm{Fe}$ & $\mathrm{Cu}$ & Au & Ag & $\mathbf{P b}$ & $\mathbf{B i}$ & $\mathrm{Sb}$ & $\mathrm{Te}$ & As & Se & $\mathrm{S}$ & Total & Formula \\
\hline 1 & Southern & & & & 84.69 & & & & & & 2.56 & 12.06 & 99.31 & $\mathrm{Ag}_{1.97}\left(\mathrm{~S}_{0.95} \mathrm{Se}_{0.08}\right)_{1.03}$ \\
\hline 2 & Southern & & & & 85.64 & & & & & & 2.36 & 12.04 & 100.04 & $\mathrm{Ag}_{1.99}\left(\mathrm{~S}_{0.94} \mathrm{Se}_{0.07}\right)_{1.01}$ \\
\hline 3 & Central & & & & 62.74 & & & & 36.39 & & & & 99.13 & $\mathrm{Ag}_{2.01} \mathrm{Te}_{0.99}$ \\
\hline 4 & Southern & & & & 63.38 & & & & & & & & 100.81 & $\mathrm{Ag}_{2.00} \mathrm{Te}_{1.00}$ \\
\hline 5 & Rusty & & & & 63.15 & & & & & & & & 99.89 & $\mathrm{Ag}_{2.01} \mathrm{Te}_{0.99}$ \\
\hline 6 & Rusty & & & 3.16 & 61.04 & & & & & & & & 100.52 & $\left(\mathrm{Ag}_{1.96} \cdot \mathrm{Au}_{0.06}\right)_{2.02} \mathrm{Te}_{0.99}$ \\
\hline 7 & Rusty & & & & 63.03 & & & & & & & & 100.31 & $\mathrm{Ag}_{2.00} \mathrm{Te}_{1.00}$ \\
\hline 8 & Rusty & & & 1.82 & 62.58 & & & & & & & & 101.34 & $\left(\mathrm{Ag}_{1.98} \cdot \mathrm{Au}_{0.03}\right)_{2.01} \mathrm{Te}_{0.99}$ \\
\hline 9 & Rusty & & & & 62.48 & & & & & & & & 99.00 & $\mathrm{Ag}_{2.01} \mathrm{Te}_{0.99}$ \\
\hline 10 & Southern & 22.22 & 3.75 & & 43.35 & & & & & & & 26.95 & 96.27 & $\mathrm{Ag}_{0.95}\left(\mathrm{Fe}_{0.94} \mathrm{Cu}_{0.14}\right)_{1.08} \mathrm{~S}_{1.98}$ \\
\hline 11 & Rusty & & & 24.68 & 42.9 & & & & & & & & 100.15 & $\mathrm{Ag}_{3.07} \mathrm{Au}_{0.97} \mathrm{Te}_{1.97}$ \\
\hline 12 & Rusty & & & 24.67 & 41.34 & & & & & & & & 98.11 & $\mathrm{Ag}_{3.03} \mathrm{Au}_{0.99} \mathrm{Te}_{1.99}$ \\
\hline 13 & Rusty & & & 24.74 & 40.47 & & & & & & & & 97.36 & $\mathrm{Ag}_{2.99} \cdot \mathrm{Au}_{1.00} \mathrm{Te}_{2.01}$ \\
\hline 14 & Hanging & & & 25.12 & 61.57 & & & & & & & 10.95 & 97.64 & $\mathrm{Ag}_{3.29} \cdot \mathrm{Au}_{0.74} \mathrm{~S}_{1.97}$ \\
\hline 15 & Southern & & & & 78.34 & & & 7.16 & & & & 11.86 & 97.36 & $\mathrm{Ag}_{10.06} \mathrm{Sb}_{0.81} \mathrm{~S}_{5.12}$ \\
\hline 16 & Southern & & & & 79.98 & & & 2.72 & & 3.68 & & 12.19 & 98.57 & $\mathrm{Ag}_{9.94}\left(\mathrm{As}_{0.66} \mathrm{Sb}_{0.30}\right)_{0.96} \mathrm{~S}_{5.10}$ \\
\hline 17 & Southern & & & & 78.25 & & & 7.67 & & & & 11.94 & 97.86 & $\mathrm{Ag}_{10.00} \mathrm{Sb}_{0.87} \mathrm{~S}_{5.13}$ \\
\hline 18 & Southern & & & & 76.99 & & & 3.97 & & 3.81 & 0.95 & 13.43 & 99.15 & $\mathrm{Ag}_{16.85}\left(\mathrm{As}_{1.20} \mathrm{Sb}_{0.77}\right)_{1.97}\left(\mathrm{~S}_{9.89} \mathrm{Se}_{0.28}\right)_{10.18}$ \\
\hline 19 & Central & & 3.24 & & 75.14 & & & 1.97 & & 5.53 & 1.01 & 13.65 & 97.30 & $\left(\mathrm{Ag}_{15.83} \mathrm{Cu}_{1.16}\right)_{16.99}\left(\mathrm{As}_{1.68} \mathrm{Sb}_{0.37}\right)_{2.05}\left(\mathrm{~S}_{9.68} \mathrm{Se}_{0.29}\right)_{9.97}$ \\
\hline 20 & Central & & 2.66 & & 73.34 & & & 9.55 & & & 1.15 & 12.99 & 97.03 & $\left(\mathrm{Ag}_{16.16} \mathrm{Cu}_{1.00}\right)_{17.16} \mathrm{Sb}_{1.86}\left(\mathrm{~S}_{9.63} \mathrm{Se}_{0.35}\right)_{9.63}$ \\
\hline 21 & Southern & & & 3.85 & 73.93 & & & 5.18 & & 2.98 & 1.33 & 12.82 & 96.24 & $\left(\mathrm{Ag}_{16.51} \mathrm{Au}_{0.47}\right)_{16.98}\left(\mathrm{Sb}_{1.02} \mathrm{As}_{0.96}\right)_{1.98}\left(\mathrm{~S}_{9.63} \mathrm{Se}_{0.41}\right)_{10.04}$ \\
\hline 22 & Rusty & & & & 5.89 & 69.12 & 12.03 & & & & & 12.56 & 99.60 & $\mathrm{Ag}_{0.98} \mathrm{Bi}_{1.03} \mathrm{~Pb}_{5.97} \mathrm{~S}_{7.02}$ \\
\hline
\end{tabular}

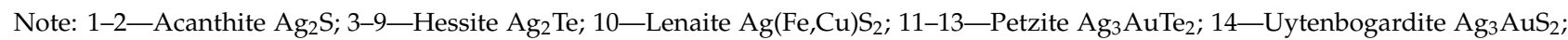
15-22-Ag-sulfosalts: $15-17-\mathrm{Ag}_{10}(\mathrm{Sb}, \mathrm{As}) \mathrm{S}_{5} ; 18-21-\mathrm{Ag}_{17}(\mathrm{Sb}, \mathrm{As})_{2}(\mathrm{~S}, \mathrm{Se})_{10} ; 22-\mathrm{AgBiPb}_{6}$.
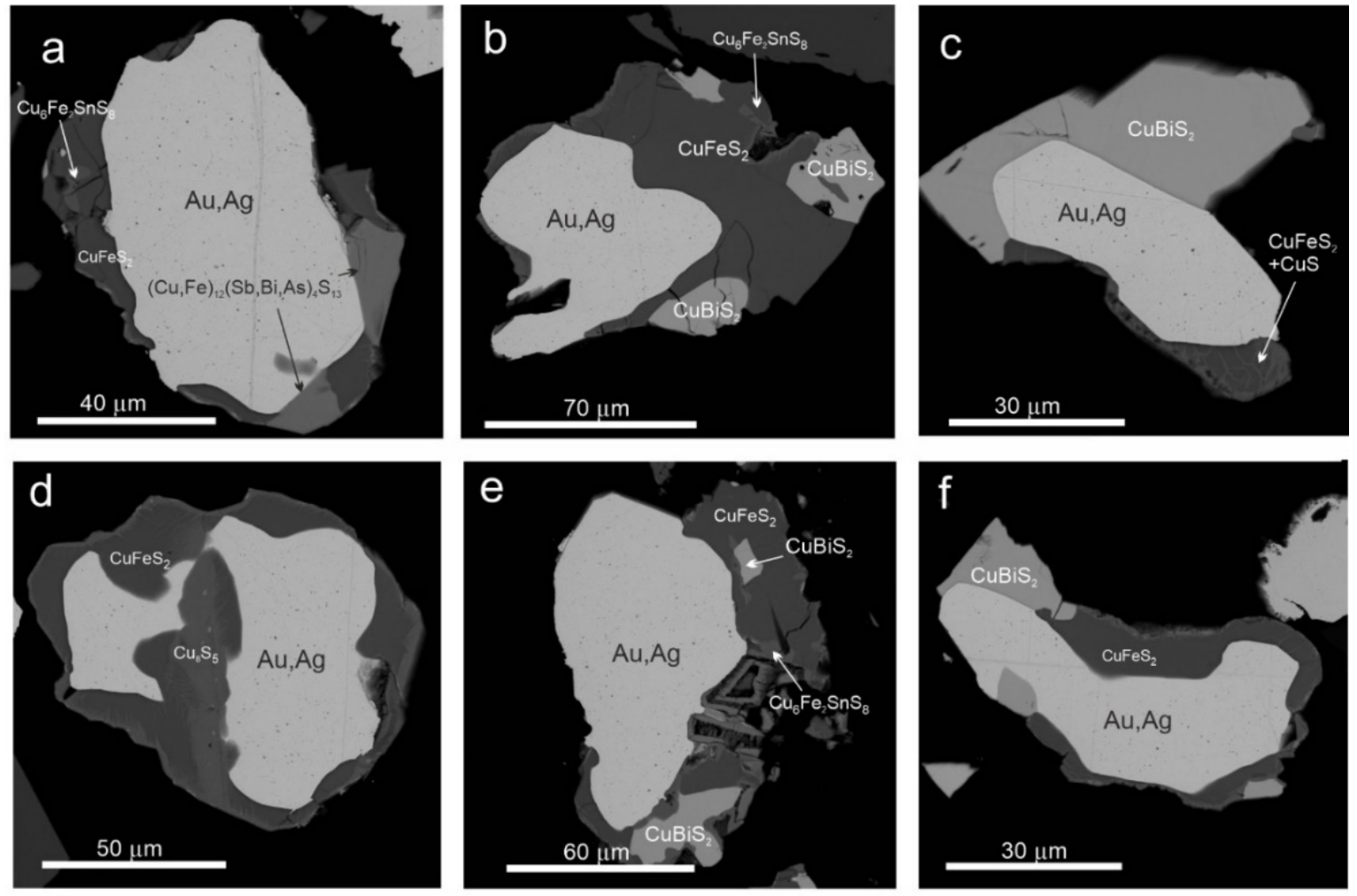

Figure 9. Au-Ag solid solutions from the gold-sulfosalt-quartz association, sample Bar-5-2, Northern zone in intergrowth with chalcopyrite $\mathrm{CuFeS}_{2}$, sometimes partially replaced by covellite $\mathrm{CuS}$ (c) or geerite $\mathrm{Cu}_{8} \mathrm{~S}_{5}(\mathrm{~d})$, mawsonite $\mathrm{Cu}_{6} \mathrm{Fe}_{2} \mathrm{SnS}_{8}$ $(\mathbf{a}, \mathbf{b}, \mathbf{e})$, emplectite $\mathrm{CuBiS}_{2}(\mathbf{b}, \mathbf{c}, \mathbf{e}, \mathbf{f})$, and bismuth-rich tetrahedrite $\mathrm{Cu}_{12}(\mathrm{Sb}, \mathrm{Bi}, \mathrm{As})_{4} \mathrm{~S}_{13}(\mathbf{a})$.

For the Northern and Hanging zones, emplectite is characterized by containing $\mathrm{Pb}$ around 2-3 wt. \% and sometimes $\mathrm{Sb}$ up to $1.81 \mathrm{wt}$. \%. Mawsonite $\mathrm{Cu}_{6} \mathrm{Fe}_{2} \mathrm{SnS}_{8}$ is also often found within this association (Figure 9a,e,g). Additionally, there is tetrahedrite of unusual composition, in which the Bi concentration either prevails over As$(\mathrm{Cu}, \mathrm{Fe}, \mathrm{Zn})_{12}(\mathrm{Sb}, \mathrm{Bi}, \mathrm{As})_{4} \mathrm{~S}_{13}$-or completely replaces it (Table 5, No. 31, 32). The tetrahedrite in Bar-5-2 sample contains more than $4 \mathrm{wt}$. \% of $\mathrm{Zn}$. Secondary minerals replacing the 
chalcopyrite are typically covellite $\mathrm{CuS}$ and geerite $\mathrm{Cu}_{8} \mathrm{~S}_{5}$ (Figure $9 \mathrm{c}-\mathrm{d}$ ). Aikinite $\mathrm{PbCuBiS}_{3}$ is as well a normal-assemblage mineral of the gold-sulfosalt-quartz association in the Northern and Hanging zones (Table 5). The gold-sulfosalt-quartz association in the Rusty zone (Figure 10) is distinguished by the presence of copper-bearing (4.93 wt. \% of $\mathrm{Cu}$ ) electrum, the composition of which corresponds to the formula $\mathrm{Au}_{0.70} \mathrm{Ag}_{0.17} \mathrm{Cu}_{0.13}$, or more simply, $\mathrm{Au}_{7} \mathrm{Ag}_{2} \mathrm{Cu}$, as well as bornite (Figure 10a,b). The tetrahedrite in Bar-2-1 sample is also Bi-rich (Table 5), but its concentration is lower than that of As. Another typomorphic Bi-rich mineral, wittichenite $\mathrm{Cu}_{3} \mathrm{BiS}_{3}$, instead of emplectite $\left(\mathrm{CuBiS}_{2}\right)$, is characteristic of this association in the Rusty zone (Figure 10e-h).
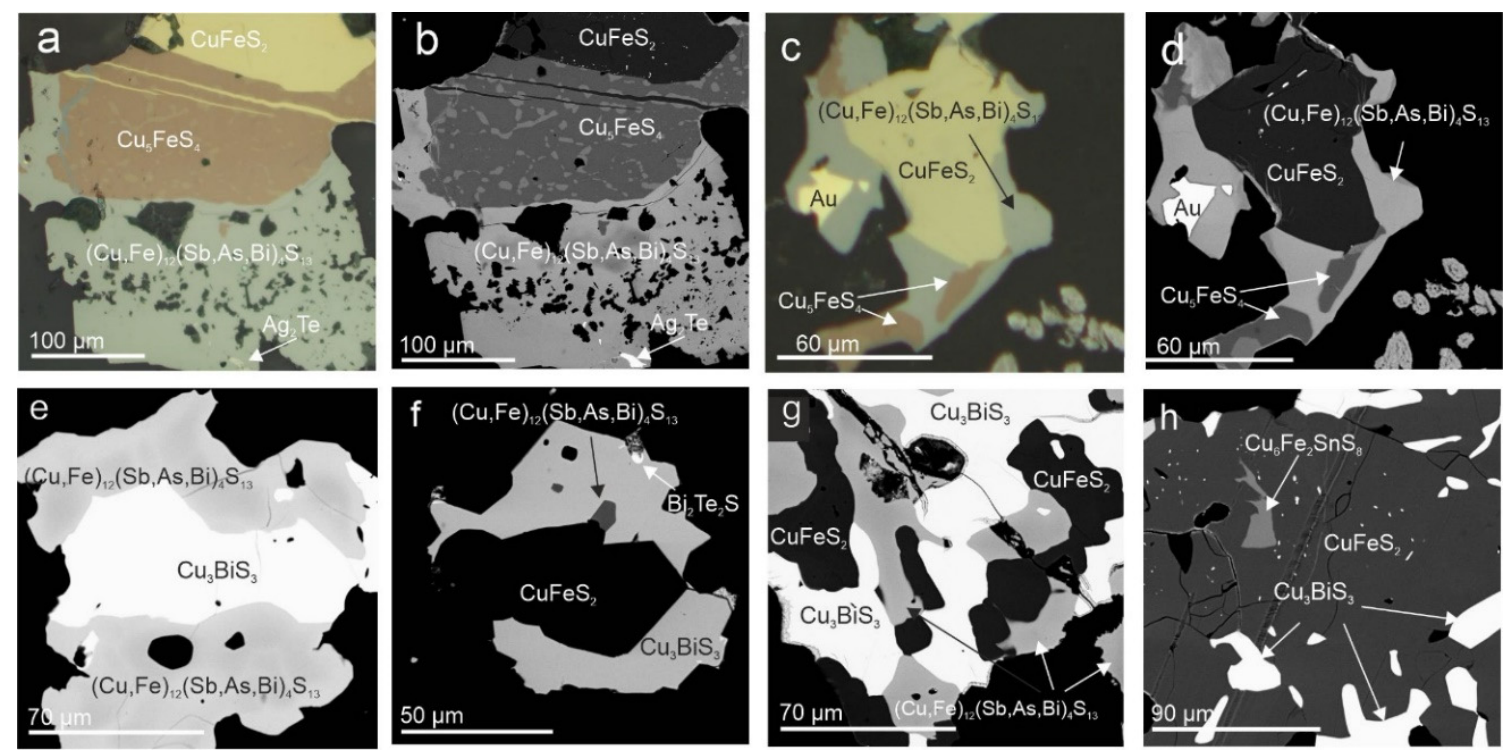

Figure 10. Microparagenesis of the gold-sulfosalt-quartz association, polished section of the Bar-2-1 sample, Rusty zone: (a,c) - in reflected light; $(\mathbf{b}, \mathbf{d}-\mathbf{h})$-SEM image. Intergrowth of chalcopyrite, bornite $\mathrm{Cu}_{5} \mathrm{FeS}_{4}$ and tetrahedrite $(\mathrm{Cu}, \mathrm{Fe})_{12}(\mathrm{Sb}, \mathrm{As}, \mathrm{Bi})_{4} \mathrm{~S}_{13}$ including the hessite $\mathrm{Ag}_{2} \mathrm{Te}$ grain; $(\mathbf{c}, \mathbf{d})-\mathrm{Cu}$-bearing electrum intergrown with chalcopyrite, bornite and tetrahedrite; (e-h) Intergrowths of chalcopyrite with sulfosalts of different composition: tetrahedrite (e-g), wittichenite $\mathrm{Cu}_{3} \mathrm{BiS}_{3}(\mathbf{e}, \mathbf{g}, \mathbf{h})$; tetradymite $\mathrm{Bi}_{2} \mathrm{Te}_{2} \mathrm{~S}(\mathbf{f})$ and mawsonite $\mathrm{Cu}_{6} \mathrm{Fe}_{2} \mathrm{SnS}_{8}$. 
Table 5. Composition of minerals from the gold-sulfosalt-quartz association, wt. \%.

\begin{tabular}{|c|c|c|c|c|c|c|c|c|c|c|c|c|c|c|c|c|}
\hline No & Sample & Zone & $\mathrm{Fe}$ & $\mathrm{Cu}$ & $\mathrm{Zn}$ & $\mathrm{Au}$ & Sn & $\mathrm{Pb}$ & $\mathbf{B i}$ & $\mathrm{Sb}$ & $\mathrm{Te}$ & As & Se & $\mathrm{s}$ & Total & Formula \\
\hline 1 & Bar-2-1 & Rusty & & & & & & 85.02 & & & & & & 12.78 & 97.80 & $\mathrm{~Pb}_{1.01} \mathrm{~S}_{0.99}$ \\
\hline 2 & Bar-2-1 & Rusty & & & & & & 85.63 & & & & & & 12.61 & 98.24 & $\mathrm{~Pb}_{1.02} \mathrm{~S}_{0.98}$ \\
\hline 3 & Bar-2-1 & Rusty & & & & 1.18 & & 84.81 & & & & & & 13.16 & 99.15 & $\left(\mathrm{~Pb}_{0.99} \mathrm{Au}_{0.01}\right)_{1.00} \mathrm{~S}_{0.99}$ \\
\hline 4 & Bar-2-1 & Rusty & & & & 2.02 & & 86.02 & & & & & & 12.83 & 100.87 & $\left(\mathrm{~Pb}_{1.01} \mathrm{Au}_{0.02}\right)_{1.03} \mathrm{~S}_{0.97}$ \\
\hline 6 & Bar-2-1 & Rusty & & & & 1.54 & & 85.81 & & & & & & 12.91 & 100.26 & $\left(\mathrm{~Pb}_{1.00} \mathrm{Fe}_{0.02} \mathrm{Au}_{0.02}\right)_{1.04} \mathrm{~S}_{0.98}$ \\
\hline 7 & Bar-5-2 & Northern & 1.62 & 73.92 & & & & & & & & & & 24.05 & 99.59 & $\left(\mathrm{Cu}_{7.79} \mathrm{Fe}_{0.19}\right)_{7.98} \mathrm{~S}_{5.02}$ \\
\hline 8 & Bar-5-2 & Northern & 3.11 & 73.65 & & & & & & & & & & 24.32 & 101.08 & $\left(\mathrm{Cu}_{7.64} \mathrm{Fe}_{0.37}\right)_{8.01} \mathrm{~S}_{5.00}$ \\
\hline 9 & Bar-5-2 & Northern & 0.60 & 74.09 & & & & & & & & & & 23.53 & 98.22 & $\left(\mathrm{Cu}_{7.93} \mathrm{Fe}_{0.07}\right)_{8.00} \mathrm{~S}_{4.99}$ \\
\hline 10 & Bar-4 & Hanging & & 99.73 & & & & & & & & & & & 99.73 & $\mathrm{Cu}_{1.00}$ \\
\hline 12 & Bar-4 & Hanging & & 64.90 & 36.09 & & & & & & & & & & 100.99 & $\mathrm{Cu}_{1.30} \mathrm{Zn}_{0.70}$ \\
\hline 13 & Bar-5-2 & Northern & & 10.46 & & & & 37.22 & 36.78 & & & & & 15.32 & 99.78 & $\mathrm{~Pb}_{1.08} \mathrm{Cu}_{0.99} \mathrm{Bi}_{1.06} \mathrm{~S}_{2.87}$ \\
\hline 14 & Bar-4 & Hanging & & 10.24 & & & & 38.39 & 36.86 & & & & & 15.23 & 100.72 & $\mathrm{~Pb}_{1.11} \mathrm{Cu}_{0.97} \mathrm{Bi}_{1.06} \mathrm{~S}_{2.86}$ \\
\hline 15 & Bar-4 & Hanging & & 10.75 & & & & 39.12 & 34.75 & & & & & 15.02 & 99.64 & $\mathrm{~Pb}_{1.14} \mathrm{Cu}_{1.02} \mathrm{Bi}_{1.00} \mathrm{~S}_{2.83}$ \\
\hline 16 & Bar-4 & Hanging & 0.41 & 10.15 & & & & 38.84 & 36.53 & & & & & 14.96 & 100.89 & $\mathrm{~Pb}_{1.13}\left(\mathrm{Cu}_{0.96} \mathrm{Fe}_{0.04}\right)_{1.00} \mathrm{Bi}_{1.05} \mathrm{~S}_{2.81}$ \\
\hline 17 & Bar-4 & Hanging & 0.27 & 18.69 & & & & 2.04 & 59.60 & & & & & 18.18 & 98.78 & $\left(\mathrm{Cu}_{1.01} \mathrm{Fe}_{0.02}\right)_{1.03}\left(\mathrm{Bi}_{0.98} \mathrm{~Pb}_{0.03}\right)_{1.01} \mathrm{~S}_{1.95}$ \\
\hline 18 & Bar-5-2 & Northern & & 18.55 & & & & 2.41 & 57.12 & 0.91 & & & & 18.33 & 97.32 & $\mathrm{Cu}_{1.01}\left(\mathrm{Bi}_{0.95} \mathrm{~Pb}_{0.04} \mathrm{Sb}_{0.03}\right)_{1.02} \mathrm{~S}_{1.98}$ \\
\hline 19 & Bar-5-2 & Northern & & 18.27 & & & & 2.90 & 57.34 & 1.27 & & & & 18.20 & 97.98 & $\mathrm{Cu}_{1.00}\left(\mathrm{Bi}_{0.95} \mathrm{~Pb}_{0.05} \mathrm{Sb}_{0.04}\right)_{1.04} \mathrm{~S}_{1.97}$ \\
\hline 20 & Bar-5-2 & Northern & & 18.31 & & & & 2.73 & 57.56 & 1.81 & & & & 18.09 & 98.50 & $\mathrm{Cu}_{1.00}\left(\mathrm{Bi}_{0.95} \mathrm{~Pb}_{0.05} \mathrm{Sb}_{0.05}\right)_{1.05} \mathrm{~S}_{1.95}$ \\
\hline 21 & Bar-5-2 & Northern & & 18.27 & & & & 3.03 & 58.64 & & & & & 17.88 & 97.82 & $\mathrm{Cu}_{1.01}\left(\mathrm{Bi}_{0.98} \mathrm{~Pb}_{0.05}\right)_{1.03} \mathrm{~S}_{1.96}$ \\
\hline 24 & Bar-5-2 & Northern & 13.01 & 43.29 & & & 13.67 & & & & & & & 29.36 & 99.33 & $\mathrm{Cu}_{5.95} \mathrm{Fe}_{2.04} \mathrm{Sn}_{1.01} \mathrm{~S}_{8.00}$ \\
\hline 25 & Bar-5-2 & Northern & 11.46 & 42.93 & 1.43 & & 13.36 & & & & & & & 28.81 & 97.99 & $\mathrm{Cu}_{6.00}\left(\mathrm{Fe}_{1.82} \mathrm{Zn}_{0.19}\right)_{2.01} \mathrm{Sn}_{1.00} \mathrm{~S}_{7.98}$ \\
\hline 26 & Bar-5-2 & Northern & 12.42 & 42.34 & & & 13.28 & & 1.84 & & & & & 28.32 & 98.20 & $\mathrm{Cu}_{5.98} \mathrm{Fe}_{2.00} \mathrm{Sn}_{1.00}\left(\mathrm{~S}_{7.93} \mathrm{Bi}_{0.08}\right)_{8.01}$ \\
\hline 27 & Bar-5-2 & Northern & 9.59 & 37.78 & 3.36 & & 17.87 & & & & & & & 28.68 & 97.28 & $\mathrm{Cu}_{7.98}\left(\mathrm{Fe}_{2.30} \mathrm{Zn}_{0.69}\right)_{2.99} \mathrm{Sn}_{2.02} \mathrm{~S}_{12.01}$ \\
\hline 28 & Bar-5-2 & Northern & 9.93 & 37.46 & 2.70 & & 17.47 & & & & 2.03 & & & 27.89 & 97.48 & $\mathrm{Cu}_{8.00}\left(\mathrm{Fe}_{2.41} \mathrm{Zn}_{0.56}\right)_{2.97} \mathrm{Sn}_{2.00}\left(\mathrm{~S}_{11.81} \mathrm{Te}_{0.22}\right)_{12.03}$ \\
\hline 29 & Bar-5-2 & Northern & & & & & & & 59.34 & & 36.16 & & 0.81 & 4.25 & 100.56 & $\mathrm{Bi}_{2.00} \mathrm{Te}_{2.00}\left(\mathrm{~S}_{0.93} \mathrm{Se}_{0.07}\right)_{1.00}$ \\
\hline 30 & Bar-5-2 & Northern & & & & & & & 58.71 & & 36.32 & & 0.91 & 4.29 & 100.23 & $\mathrm{Bi}_{1.98} \mathrm{Te}_{2.00}\left(\mathrm{~S}_{0.97} \mathrm{Se}_{0.08}\right)_{1.02}$ \\
\hline 31 & Bar-5-2 & Northern & 1.66 & 38.44 & 4.37 & & & & 10.40 & 18.19 & & 2.70 & & 24.33 & 100.09 & $\left(\mathrm{Cu}_{10.35} \mathrm{Zn}_{1.14} \mathrm{Fe}_{0.51}\right)_{12.00}\left(\mathrm{Sb}_{2.56} \mathrm{Bi}_{0.85} \mathrm{As}_{0.62}\right)_{4.03} \mathrm{~S}_{12.98}$ \\
\hline 32 & Bar-5-2 & Northern & 1.76 & 36.73 & 4.41 & & & 1.83 & 9.81 & 20.88 & & & & 23.62 & 99.04 & $\left(\mathrm{Cu}_{10.21} \mathrm{Zn}_{1.19} \mathrm{Fe}_{0.56}\right)_{11.96}\left(\mathrm{Sb}_{3.03} \mathrm{Bi}_{0.16}\right)_{4.02} \mathrm{~S}_{13.02}$ \\
\hline 33 & Bar-2-1 & Rusty & 3.94 & 41.89 & & & & & & 25.14 & & 3.56 & & 24.95 & 99.48 & $\left(\mathrm{Cu}_{10.85} \mathrm{Fe}_{1.16}\right)_{12.01}\left(\mathrm{Sb}_{3.40} \mathrm{As}_{0.78}\right)_{4.18} \mathrm{~S}_{12.81}$ \\
\hline 34 & Bar-2-1 & Rusty & 3.77 & 41.76 & & & & & 3.31 & 23.86 & & 2.47 & & 25.02 & 100.19 & $\left(\mathrm{Cu}_{10.89} \mathrm{Fe}_{1.12}\right)_{12.01}\left(\mathrm{Sb}_{3.25} \mathrm{As}_{0.55} \mathrm{Bi}_{0.26}\right)_{4.18} \mathrm{~S}_{12.93}$ \\
\hline 35 & Bar-2-1 & Rusty & 4.29 & 41.93 & & & & & 3.37 & 24.09 & & 2.70 & & 25.03 & 101.41 & $\left(\mathrm{Cu}_{10.83} \mathrm{Fe}_{1.26}\right)_{12.09}\left(\mathrm{Sb}_{3.25} \mathrm{As}_{0.59} \mathrm{Bi}_{0.26}\right)_{4.10} \mathrm{~S}_{12.81}$ \\
\hline 36 & Bar-2-1 & Rusty & 3.69 & 39.43 & & & & & 9.04 & 19.69 & & 2.16 & & 23.74 & 97.75 & $\left(\mathrm{Cu}_{10.83} \mathrm{Fe}_{1.15}\right)_{11.99}\left(\mathrm{Sb}_{2.82} \mathrm{As}_{0.50} \mathrm{Bi}_{0.76}\right)_{4.08} \mathrm{~S}_{12.93}$ \\
\hline 37 & Bar-2-1 & Rusty & 3.20 & 39.71 & & & & & 13.1 & 16.36 & & 2.79 & & 23.59 & 98.75 & $\left(\mathrm{Cu}_{10.97} \mathrm{Fe}_{1.01}\right)_{11.98}\left(\mathrm{Sb}_{2.36} \mathrm{Bi}_{1.10} \mathrm{As}_{0.65}\right)_{4.11} \mathrm{~S}_{12.91}$ \\
\hline 38 & Bar-2-1 & Rusty & 3.75 & 39.26 & & & & & 7.90 & 16.13 & & 4.29 & & 23.86 & $96.00 *$ & $\left(\mathrm{Cu}_{10.77} \mathrm{Fe}_{1.17} \mathrm{Ag}_{0,13}\right)_{12.07}\left(\mathrm{Sb}_{2.31} \mathrm{As}_{1.00} \mathrm{Bi}_{0.66}\right)_{3.97} \mathrm{~S}_{12.97}$ \\
\hline 39 & Bar-2-1 & Rusty & 3.92 & 39.46 & & & & & 9.20 & 15.98 & & 4.50 & & 24.1 & $97.88^{* *}$ & $\left(\mathrm{Cu}_{10.69} \mathrm{Fe}_{1.21} \mathrm{Ag}_{0,11}\right)_{12.01}\left(\mathrm{Sb}_{2.26} \mathrm{As}_{1.03} \mathrm{Bi}_{0.76}\right)_{4.05} \mathrm{~S}_{12.94}$ \\
\hline 40 & Bar-2-1 & Rusty & 4.30 & 40.83 & & & & & 9.10 & 14.59 & & 5.85 & & 24.76 & 99.43 & $\left(\mathrm{Cu}_{10.75} \mathrm{Fe}_{1.29}\right)_{12.04}\left(\mathrm{Sb}_{2.01} \mathrm{As}_{1.31} \mathrm{Bi}_{0.73}\right)_{4.04} \mathrm{~S}_{12.92}$ \\
\hline 41 & Bar-2-1 & Rusty & 4.47 & 41.28 & & & & & 10.58 & 9.93 & & 7.97 & & 25.28 & 99.51 & $\left(\mathrm{Cu}_{10.72} \mathrm{Fe}_{1.32}\right)_{12.05}\left(\mathrm{Sb}_{1.35} \mathrm{As}_{1.76} \mathrm{Bi}_{0.84}\right)_{3.94} \mathrm{~S}_{13.02}$ \\
\hline
\end{tabular}


Table 5. Cont.

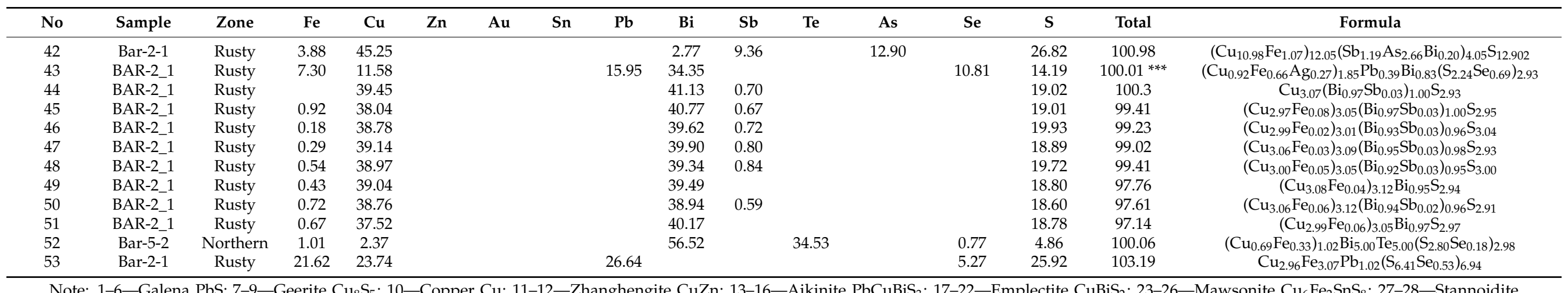

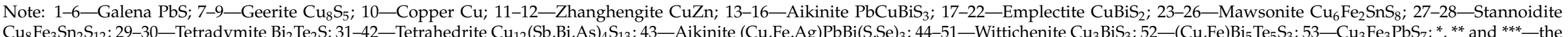
totals include $0.82,0.72$ and $5.83 \mathrm{wt}$ \% of $\mathrm{Ag}$, respectively. 


\subsection{Concentration of Ore and Chalcogenic Elements}

The concentration of ore and chalcogenic elements in samples from different zones of the Baranyevskoye deposit were obtained. The concentration of precious metals is unevenly distributed: tenths of ppm Au in the Southern zone, units of ppm in the Rusty and Central zones, tens of ppm in the Hanging zone, whereas the highest concentrations of hundreds of ppm occur in the Northern zone (Table 6). In general, Au prevails over Ag in all zones $(\mathrm{Au} / \mathrm{Ag}$ ratios are 2.80-9.91 and $\mathrm{Ag} / \mathrm{Au}$ ratios are 0.10-3.72) except for the Southern zone, in which the $\mathrm{Au} / \mathrm{Ag}$ ratios vary in the range of $0.16-0.52$ for the quartz vein, and reaches up to 2.35 in the metasomatitic host rock (sample BAR-6-5). Thus, the samples from the tunnels (BAR-5) of the Northern Zone are the richest in Au. Sample BAR-5-1 represents the gold-pyrite-quartz association, while sample BAR-5-2 represents the gold-sulfosalt-quartz association, in which the concentrations of $\mathrm{Pb}, \mathrm{Sb}$, Se are increased by one order of magnitude, $\mathrm{Cu}$ and $\mathrm{Sn}$ by two orders of magnitude, and Bi by four orders of magnitude compared with the content of these elements in samples from other zones. At the same time, the As concentration is not high compared to its anomalous values (1030 ppm) in the gold-pyrite-quartz association of the same zone. The aforementioned distribution of elements is consistent with mineralogical features: As-bearing pyrite in one association and the presence of many Bi-rich and Sn-bearing sulfosalts included in chalcopyrite in another.

Table 6. Concentration of ore and chalcogenic elements in rocks of different zones of the Baranyevskoe deposit, ppm.

\begin{tabular}{|c|c|c|c|c|c|c|c|c|c|c|c|c|c|}
\hline Zone & Sample & $\mathrm{Au}$ & Ag & $\mathrm{Au} / \mathrm{Ag}$ & $\mathrm{Cu}$ & As & $\mathrm{Bi}$ & $\mathrm{Pb}$ & $\mathrm{Sb}$ & Se & Sn & $\mathrm{Zn}$ & $\mathrm{Te}$ \\
\hline Rusty & Bar-2-1 & 3.77 & 0.98 & 3.85 & 20.40 & 24 & 0.50 & 19.90 & 13.30 & $<2$ & $<0.30$ & 22 & 0.49 \\
\hline Central & Bar-3 & 6.78 & 1.96 & 3.46 & 88.90 & 21 & 0.21 & 56.40 & 23.30 & $<2$ & 0.30 & 95 & 0.22 \\
\hline Central & Bar-3-1 & 6.47 & 2.31 & 2.80 & 69.00 & 152 & 0.14 & 67.30 & 26.40 & $<2$ & $<0.30$ & 67 & 0.12 \\
\hline Hanging & Bar-4 & 43.70 & 14.10 & 3.10 & 24.30 & 42 & 0.40 & 35.40 & 11.60 & $<2$ & 0.30 & 66 & 0.28 \\
\hline Northern & Bar-5-1 & 135 & 37.60 & 3.59 & 80.90 & 1030 & 0.27 & 21.30 & 56.70 & $<2$ & 0.40 & 22 & 0.10 \\
\hline Northern & Bar-5-2 & 110 & 11.10 & 9.91 & 3240 & 68 & 7800 & 274 & 169 & 36 & 116 & 15 & 21.6 \\
\hline Southern & Bar-6-1 & 0.22 & 1.34 & 0.16 & 88.10 & 11 & 0.51 & 26.70 & 17.00 & $<2$ & $<0.30$ & 39 & 0.89 \\
\hline Southern & Bar-6-2 & 0.16 & 0.43 & 0.37 & 128 & 38 & 0.16 & 37.70 & 14.10 & $<2$ & 0.40 & 75 & 0.41 \\
\hline Southern & Bar-6-3 & 0.36 & 1.34 & 0.27 & 14.10 & 19 & 0.05 & 50.70 & 9.56 & $<2$ & 0.40 & 43 & 0.65 \\
\hline Southern & Bar-6-4 & 0.16 & 0.31 & 0.52 & 34.70 & 54 & 0.09 & 25.00 & 6.83 & $<2$ & 0.70 & 78 & 0.93 \\
\hline Southern & Bar-6-5 & 0.68 & 0.29 & 2.35 & 25.80 & 39 & 0.04 & 12.50 & 12.90 & $<2$ & 0.80 & 76 & 0.10 \\
\hline
\end{tabular}

\subsection{Study on Fluid Inclusions}

Fluid inclusions were investigated only for the gold-sulfosalt-quartz association. Small (15-20 $\mu \mathrm{m})$ primary gaseous and two-phase gas-liquid inclusions contained in quartz were identified. In the central parts of translucent crystals of quartz, there are mainly groups of isometric two-phase inclusions (Figure 11a) whereas gas and two-phase fluid inclusions, on the growth zones of quartz crystals, have an irregular shape (Figure 11b).

Homogenization temperature of fluid inclusions varied in the range of $226-298{ }^{\circ} \mathrm{C}$ (Figure 12a). The ice melting in cooled fluid inclusions appeared at temperatures from -0.8 to $-0.2^{\circ} \mathrm{C}$ (Table 7 ). The salinity of fluid inclusions was estimated from 0.4 to $1.2 \mathrm{wt}$. $\%$ $\mathrm{NaCl}$ eq. Melting of eutectic in frozen fluid inclusions occurred in the temperature range of -49 to $-25{ }^{\circ} \mathrm{C}$. 

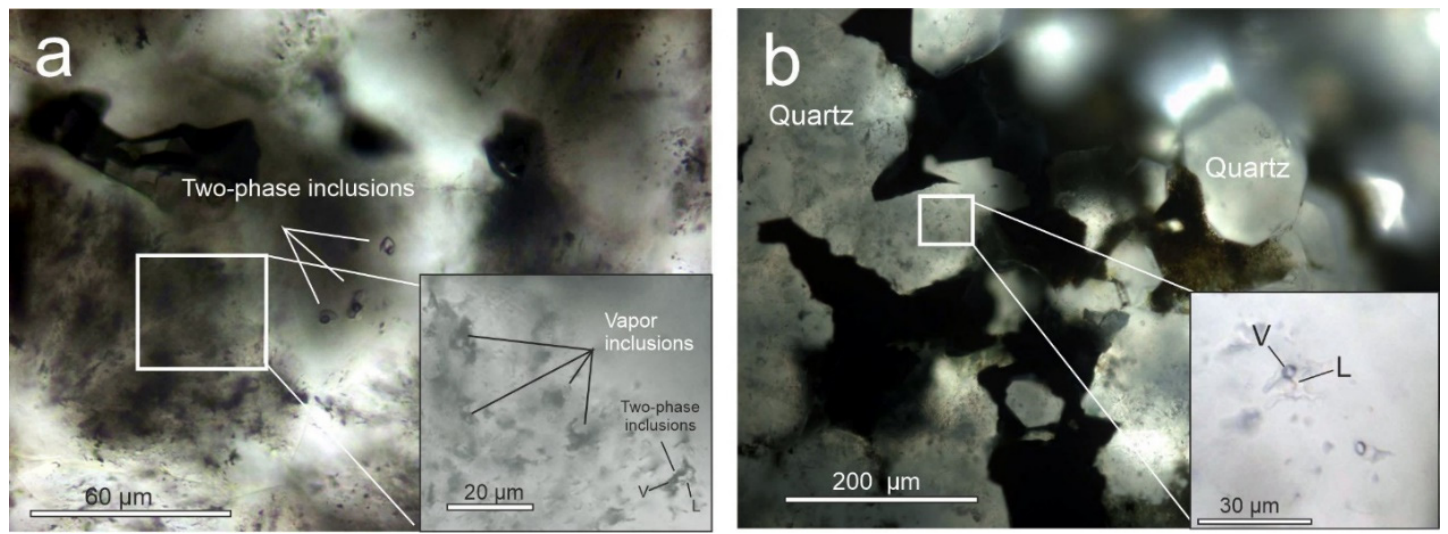

Figure 11. Microphotographs of quartz from the gold sulfosalt-quartz association: (a)—two-phase inclusions of isometric shape in the center of a transparent quartz crystal (sample Bar-5_2/1). Gaseous and two-phase inclusions of irregular shape on the crystal growth zone are shown in the inset; (b) - paragenesis of quartz, chalcopyrite and sulfosalts (dark) in a quartz vein (sample Bar-5_2/1). Primary two-phase fluid inclusions in a quartz crystal are shown in the inset.
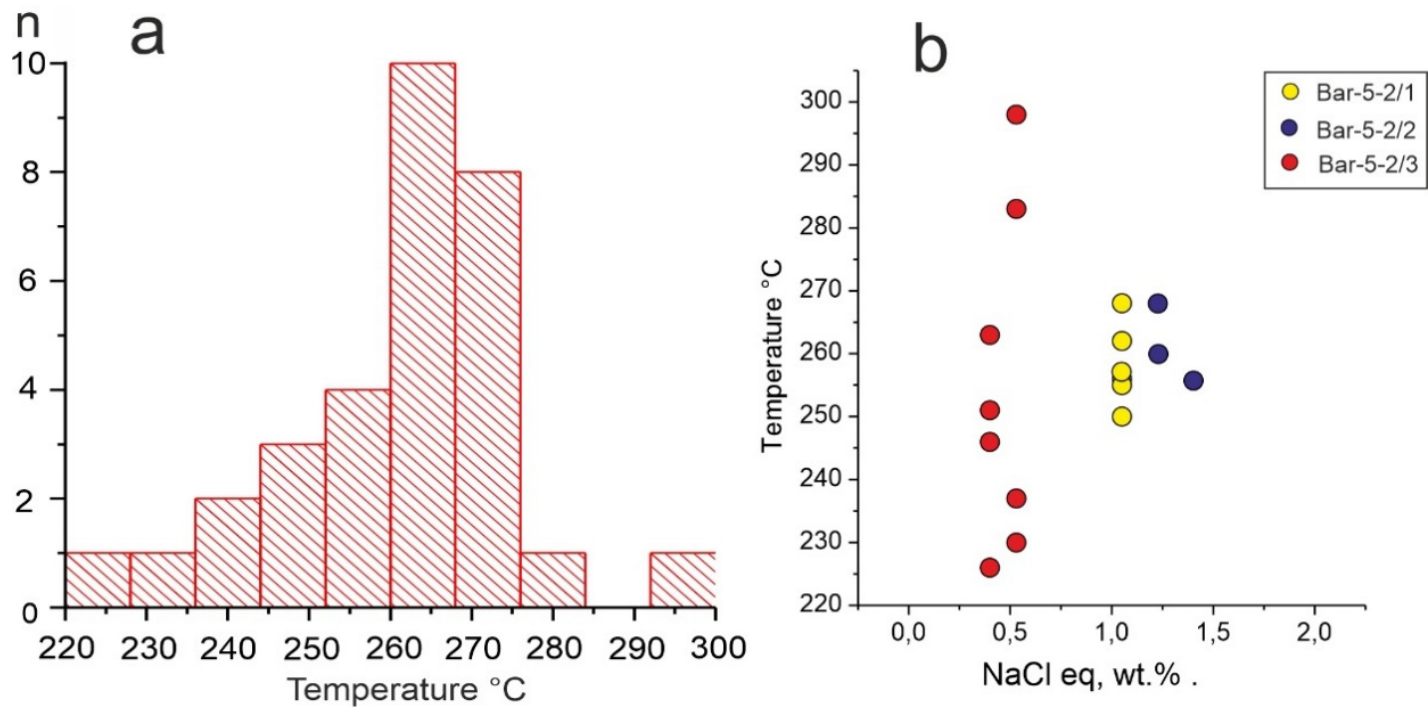

Figure 12. Results of the fluid inclusion study: (a) histogram of homogenization temperatures of fluid inclusions in quartz of the gold-sulfosalt-quartz association from the Northern Zone of the Baranyevskoe deposit; (b) temperature of homogenization of inclusions versus their salinity. Bar-5-2/1 to Bar-5-2/3 are different sample Bar-5-2 areas of the gold-sulfosalt-quartz association.

Table 7. Summary of the microthermometric study of fluid inclusions in quartz from the gold-sulfosalt-quartz association.

\begin{tabular}{ccccccc}
\hline Sample & $\boldsymbol{n}$ & $\mathbf{T h}{ }^{\circ} \mathbf{C}$ & Teu $^{\circ} \mathbf{C}$ & Tm Ice $^{\circ} \mathbf{C}$ & Salinity, wt. \% NaCl eq. & MPa \\
\hline Bar-5 2/1 & 6 & $268-250$ & -45 & $-0.6 \ldots-0.8$ & $1.2-1.1$ & $5.3-3.9$ \\
Bar-5 2/2 & 13 & $268-256$ & -49 & -0.7 & 1.2 & $5.3-4.4$ \\
Bar-5 2/3 & 11 & $298-226$ & -27 & $-0.2 \ldots-0.4$ & $0.5-0.4$ & $2.6-8.3$ \\
\hline
\end{tabular}

Note: $n$-number of analyses; $\mathrm{Th}{ }^{\circ} \mathrm{C}$-homogenization temperature of fluid inclusions; Teu ${ }^{\circ} \mathrm{C}$ - eutectic temperature (first melting temperature); $\mathrm{Tm}$ ice ${ }^{\circ} \mathrm{C}$ - final ice melting temperature; $\mathrm{MPa}$ - calculated pressure [22]; eq-equivalent.

Fluid inclusions in quartz from the gold-sulfosalt quartz association are characterized by eutectic melting at range of -45 to $-27^{\circ} \mathrm{C}$. The fluid inclusions in the quartz of this ore association contain $\mathrm{NaCl}$ and $\mathrm{KCl}$. Low melting temperatures of eutectics from -49 to $-27 \mathrm{C}^{\circ}$ are characteristic of fluid inclusions in quartz of the gold-sulfosalt-quartz association with high-grade gold (Table 7). With regard to the composition of fluids at the latest stage, in addition to $\mathrm{NaCl}$, there could be admixtures of $\mathrm{CaCl}_{2}, \mathrm{MgCl}_{2}, \mathrm{FeCl}_{2}, \mathrm{FeCl}_{3}$, 
$\mathrm{K}_{2} \mathrm{CO}_{3}$, which may lower the melting point of the eutectic of fluid inclusions [24]. The composition of gas inclusions, apparently, is dominated by water vapor.

The wide range of temperature of homogenization in the fluid inclusions $\left(298-226^{\circ} \mathrm{C}\right)$ along with a narrow range of salinity $(0.4-1.2 \mathrm{wt} . \% \mathrm{NaCl}$ eq.) (Figure $12 \mathrm{~b}$ ) may be explained by processes of mixing of hot magmatogenic fluids with cold fresh meteoric waters. The presence of primary gaseous and two-phase fluid inclusions on the growth zones of quartz crystals indicates boiling of the ore-forming fluid. The calculated pressure of homogenization of fluid inclusions in quartz from the late stage of mineralization of the Baranyevskoe deposit varies from 2.6 to $8.3 \mathrm{MPa}$, and it is mostly close to the pressures of ore formation in the near-surface volcanic-hydrothermal mineral systems. If we take these values for hydrostatic pressure, then we can assume that these ores from the Baranyevskoe deposit were formed at a depth of 260 to $530 \mathrm{~m}$.

The temperature and salinity of ore-forming fluids of the late gold-sulfosalt-quartz association varied over a narrow interval; however, an even more complex composition of salts in the ore-forming fluids is assumed at the final stage of gold-sulfosalt-quartz associations: salts that may be expected to be present: $\mathrm{NaCl}, \mathrm{CaCl}_{2}, \mathrm{MgCl}_{2}, \mathrm{FeCl}_{2}, \mathrm{FeCl}_{3}$, $\mathrm{K}_{2} \mathrm{CO}_{3}$, compared to the composition of fluids $(\mathrm{NaCl}$ and $\mathrm{KCl})$ at the initial stage of formation of this association.

\section{Discussion}

Gold from the Baranyevskoye deposit is subdivided into two classes: the first includes a series of compositions related to low-grade gold and electrum with a fineness of (52-74 at. \% Au) (Figure 13). Sufficiently large grains (50-100 microns) are, as a rule, in association with pyrite and are characteristic of the early high-temperature stage. Similar compositions have been described for hypogenic gold at the Aginskoye deposit [11]. The second class corresponds to high-grade gold (88-94 at. \% Au) (Figure 13), which, together with chalcopyrite and Bi-rich sulfosalts, compose the mineral association of the lowertemperature stage. However, it should be noted that high-grade gold is also rarely found in the early gold-pyrite-quartz association, being represented there only by thin worm-like veins included in low-grade gold (Figure 8g).

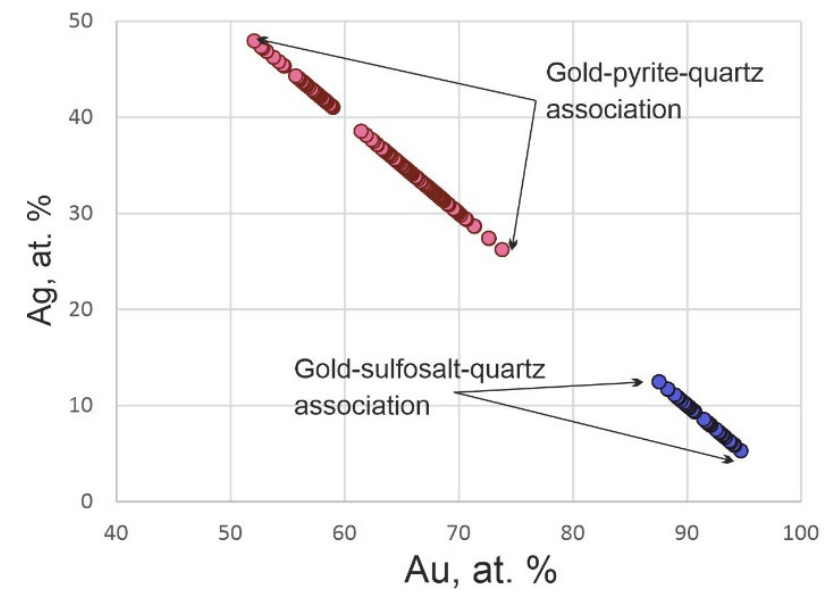

Figure 13. Compositions of Au-Ag alloys (at. \%) in both associations of the Baranyevskoye deposit.

Similar textures were previously described at the Aginskoye deposit [11]. According to the authors, such high-grade gold is secondary in origin, formed during the oxidation of hypogenic gold by meteoric waters at the stage of hypergenesis. However, we consider that the formation of high-grade gold in the Baranyevskoe deposit is mainly due to a change in the physicochemical conditions and the composition of hydrothermal solutions. Andreeva and Kudaeva [20], who previously studied the typomorphism of gold in the Balkhach ore cluster, noted that the grain size and fineness increase within the transition from quartz-carbonate-adularia stockwork rocks to carbonate rocks and quartz-carbonate- 
adularia veins. On the other hand, silver content in gold is considered as an indicator of the temperature regime during the formation of ores [31]. The composition of native gold evolves from very high-grade gold to electrum and Hg-bearing gold in the Western Tuva deposits (Russia) [32] as opposed to what is described in this study. Electrum is associated with pyrite at the Valunistoye deposit (Chukotka), and high-grade gold is found in the form of rims and veins in electrum, occurring later in the paragenesis [33], as in our case. The $\mathrm{Au}-\mathrm{Ag}-\mathrm{Cu}$ system with reference to [34] is shown in [35]. Composition-temperature ranges in this isotherm reflect metastable equilibria. On one side of the triangle, there is a wide row of Au-Ag solid solutions below $300^{\circ} \mathrm{C}$ isotherm. That is, alloys of different compositions can crystallize at the same temperature. Therefore, at temperatures corresponding to hydrothermal conditions, opposite trends of the variation in Ag content, in gold grains, are observed for different deposits.

Pyrite is one of the most important indicator minerals in studying the features of the genesis in ore deposits. The As concentration in the pyrite of the Baranyevskoe deposit (7.37 wt. \%) exceeds the As in pyrite of numerous deposits, including the values in the Kumroch deposit, where pyrite contains up to $6.79 \mathrm{wt}$. \% As [36]. For instance, the content of As in pyrite at the Zaozigou Gold Deposit (Central China) is $4.1 \mathrm{wt}$. \% [37], it is $4.5 \mathrm{wt}$. \% in pyrite of the Roudný deposit, Bohemian Massif [38], while As in pyrite from El Valle gold deposit (Spain) is the most abundant (up to $9.5 \mathrm{wt}$. \% As) [39]. Therefore, it becomes evident that the solid solution of $\mathrm{Au}$ is dominated by arsenian pyrite in all these deposits. However, high As concentrations are characteristic only for certain areas of zoned pyrite. Compositional zoning of pyrites in gold deposits reflects the chemical evolution of ore bearing fluids. The relatively high activity of As and Au during crystallization of the early generation of pyrite allowed As-bearing pyrite to be precipitated as a consequence. This is consistent with the extremely high As concentration in the samples of the goldpyrite-quartz association in the Northern Zone (Table 6). The As-bearing pyrites were formed at temperatures of at least $320-330^{\circ} \mathrm{C}$, based on arsenopyrite thermometers and fluid inclusion data [38]. The variable amount of As in grains of pyrite reflects changes in physicochemical conditions $\left(\mathrm{T}, f \mathrm{~S}_{2}, f \mathrm{O}_{2}, \mathrm{pH}\right)$ and the composition of fluids, which, at the same time, determine the appearance of a concentration gradient on the pyrite growth surface [40]. Pyrite and gold of low-grade composition in the early association are genetically linked with the Ag-Au minerals: acanthite, hessite, lenaite, petzite, utenbogardtite and $\mathrm{Ag}$-sulfosalts: $\mathrm{Ag}_{10}(\mathrm{Sb}, \mathrm{As}) \mathrm{S}_{5}$ and $\mathrm{Ag}_{17}(\mathrm{Sb}, \mathrm{As})_{2}(\mathrm{~S}, \mathrm{Se})_{10}$.

An increase in copper concentration during the development of the ore-forming system led to the formation of a later gold ore association with the leading role of chalcopyrite, as well as other cuprous phases (bornite, chalcocite, geerite, native copper and $\mathrm{Cu}-\mathrm{Zn}$ solid solutions). Simultaneously, there was an increase in the fineness of gold from 700-800\% in the early association up to $900-950 \% 00$ in the late one, as well as an increase in the concentration of bismuth (leading to the formation of emplectite $\mathrm{CuBiS}_{2}$ or wittichenite $\left.\mathrm{Cu}_{3} \mathrm{BiS}_{3}\right)$; the concentration of tin in the ore-forming system was also increased, forming mawsonite $\mathrm{Cu}_{6} \mathrm{Fe}_{2} \mathrm{SnS}_{8}$.

Primary galena from hydrothermal deposits has been shown to contain anomalous and significant levels of $\mathrm{Bi}, \mathrm{Ag}, \mathrm{Te}, \mathrm{Se}, \mathrm{Sb}, \mathrm{Cu}, \mathrm{Tl}$ and $\mathrm{Zn}$ [41]. Increased concentrations of $\mathrm{Bi}$ and $\mathrm{Ag}$ indicate the dissolution of the proportion of $\mathrm{AgBiS}_{2}$ in galena [42]. At the Baranyevskoe deposit, galena contains only traces of $\mathrm{Au}$ (up to $2.02 \mathrm{wt}$ \%), and no other minor elements were found. It is assumed that a gold-bearing variety of galena contains probably microscopic or nano-inclusions of gold or gold-bearing-minerals (https: / www. mindat.org/min-26564.html, 11 October 2021). Au-bearing galena from the Baranyevskoe deposit is, however, found as inclusions in Au-Ag alloys. Then, it could be argued that gold content was measured from the matrix during the analysis. Firstly, however, the galena inclusions are large enough (10-20 microns) for correct analysis; secondly, they look absolutely homogeneous (Figure 8b); and thirdly, Ag is absent in the analysis of galena, and it should have been captured together with Au. Therefore, Au-containing galena is a feature of the gold-pyrite-quartz association. 
The tetrahedrite $\left(\mathrm{Cu}_{10}(\mathrm{Fe}, \mathrm{Zn})_{2} \mathrm{Sb}_{4} \mathrm{~S}_{13}\right)$-tennantite $\left(\mathrm{Cu}_{10}(\mathrm{Fe}, \mathrm{Zn})_{2} \mathrm{As}_{4} \mathrm{~S}_{13}\right)$ solid-solution series (fahlore) is common, and widespread in Au-Ag epithermal ore deposits around the world [43]. The role of sulfosalts in gold deposits is significant, since they are closely associated with native gold, and their study is considered to be very important in identifying the nature of gold mineralization. The rather significant variability in composition makes fahlores a useful indicator of ore-forming processes and fluid compositions during their development [44].

Tetrahedrite of the Baranyevskoe deposit contains significant amounts of bismuth, which in some cases may dominate over arsenic $\mathrm{Cu}_{12}(\mathrm{Sb}, \mathrm{Bi}, \mathrm{As})_{4} \mathrm{~S}_{13}$. A similar and even richer in bismuth (up to 22.17 wt. \% Bi) tetrahedrite was described earlier in Schwarzwald ore district with 1.83 atoms per formula unit (apfu) Bi [44] compared to Baranievskoye, where $0.20-1.10$ apfu $\mathrm{Bi}$, based on 4 total apfu $(\mathrm{Sb}+\mathrm{As}+\mathrm{Bi})$ was established. However, the Birichest sulfosalts are also $\mathrm{Pb}$-bearing [45], whereas, in the herein investigated association, it lacks $\mathrm{Pb}$-Bi fahlores. Only one grain of sulfosalt containing lead $\left(\mathrm{Cu}_{3} \mathrm{Fe}_{3} \mathrm{PbS}_{7}\right)$ was found. A feature of the gold-sulfosalt-quartz association of the Baranyevskoe deposit is the presence of the Te-free tetrahedrite-tennantite series, while Te-rich fahlore (goldfieldite) is characteristic in numerous epithermal deposits [46], including the gold-forming stages of the Ozernovskoe and Aginskoe epithermal deposits in Kamchatka. Moreover, at the Aginskoe deposit, the Te-rich minerals $\left(\mathrm{AuTe}_{2}, \mathrm{PbTe}, \mathrm{Ag}_{2} \mathrm{Te}, \mathrm{Ag}_{3} \mathrm{AuTe}_{2}\right)$ are rather common, while pyrite is subordinate $[8,9,11]$. The presence of Bi-rich and, simultaneously, Te-poor varieties of sulfosalts is considered a typomorphic feature of the gold-sulfosalt-quartz association.

The increasing role of bismuth at the late gold stage (gold-sulfosalt-quartz association) expressed by the crystallization of bismuth-rich minerals-emplectite $\mathrm{CuBiS}_{2}$, wittichenite $\mathrm{Cu}_{3} \mathrm{BiS}_{3}$, tetradymite $\mathrm{Bi}_{2} \mathrm{Te}_{2} \mathrm{~S}$, sulfosalt $(\mathrm{Cu}, \mathrm{Fe}) \mathrm{Bi}_{5} \mathrm{Te}_{5} \mathrm{~S}_{3}$-is consistent with the data of the chemical analysis of the ores from this association (Table 6). Sulfosalts of copper, iron and tin-mawsonite $\mathrm{Cu}_{6} \mathrm{Fe}_{2} \mathrm{SnS}_{8}$ and stannoidite $\mathrm{Cu}_{8} \mathrm{Fe}_{3} \mathrm{Sn}_{2} \mathrm{~S}_{12}$ - are also characteristic of the gold-sulfosalt-quartz association. These minerals indicate some enrichment in tin at a late stage in the development of the ore-forming system. Mawsonite $\mathrm{Cu}_{6} \mathrm{Fe}_{2} \mathrm{SnS}_{8}$ has been identified in association with pyrite and tetrahedrite in a vein orebody, and its formation is related to interactions during the substitution of the tin-bearing famatinite by tetrahedrite [47]. The gold-sulfosalt-quartz association identified in this study is in many respects similar to that from the Kairagach gold deposit, Uzbekistan, which is characterized by a Au-Sn-Bi-Se-Te geochemical profile; namely, it is comparable to the third generation of ore mineralization of Kairagach gold deposit: Bi-sulfosalts as well as native gold of high fineness, tetrahedrite-annivite series. These are characterized by high (up to 9 wt. \%) content of $\mathrm{Bi}[48]$.

The microthermometry study on fluid inclusions in quartz with the most abundant dissemination of chalcopyrite and sulfosalts grains (gold-sulfosalts-quartz association) revealed in the quartz aggregate, temperatures corresponding to a range of $299-226{ }^{\circ} \mathrm{C}$. Similar temperatures of homogenization of primary inclusions were reported for the quartz of the Aginskoe deposit (LS type) $230-280{ }^{\circ} \mathrm{C}$ [9], and were also established for the Rodnikovoe and Asachinskoe deposits in South Kamchatka $[6,49,50]$. In addition, at the IS type field (Cesme Hafez, Iran) [51], homogenization temperatures of primary inclusions are within the same range $\left(140-280^{\circ} \mathrm{C}\right)$. At the same time, it is well compatible with many HS type deposits, e.g., Maletoyvayam $\left(255-245^{\circ} \mathrm{C}\right)$ [15].

The salinity range can be strongly influenced, on one hand, by mixing with meteoric water (dilution), or, on the other hand, by boil-off (concentrating) [52,53]. In general, the salinity of LS type deposits shows a wide range of values. The mineralization in LS type crystallizes, as a rule, from relatively dilute brines $<5 \mathrm{wt} . \% \mathrm{NaCl}$ eq. Aginskoye deposit is no greater than 2 wt. \% NaCl equiv. [9], as in the Juliet field (LS type), at the OkhotskChukotka volcanic belt, the salinity of the inclusions is around 1.2-5.6 wt. \% $\mathrm{NaCl}$ eq. [54]. However, data from fluid inclusions, in quartz, associated with the main gold stage in HS type deposits-Mt Carlton, Lepanto, Agan, Mt Carlton, NE Australia, Danchenkovskoe, and Maletoyvayam [15,55-58] - similarly indicate salinities up to $4.5 \mathrm{wt}$. $\% \mathrm{NaCl}$ eq. In 
this respect, it becomes clear that the salinity of fluid inclusions is not necessarily related to the type of deposit, but rather depends on the conditions of ore deposition [15].

Metallogenic specialization and $\mathrm{Au} / \mathrm{Ag}$ Index of ore is fundamentally related to the host environment in which ore is formed [59]. High values of this index $(<50)$ are typical for deposits related to oceanic island arcs: Central Kamchatka-1.7; Fiji-4.9; Solomon Islands-2.6. The high ratios of $\mathrm{Au} / \mathrm{Ag}(2.80-9.90)$, which characterize the Baraneyvskoe deposit, are a promising economic feature in predicting epithermal gold deposits [59].

\section{Conclusions}

Two Au-forming associations are characteristic of the Baranyevskoye deposit:

1. Early gold-pyrite-quartz, characterized by the presence of low-grade gold and electrum (660-820\% 00 ) in association with pyrite, the grains of which often exhibit oscillatory zoning with As concentrations up to $7.37 \mathrm{wt}$. \% in some areas. Accessory Au-Ag minerals: acanthite $\mathrm{AgS}_{2}$, hessite $\mathrm{AgTe}_{2}$, lenaite $\mathrm{Ag}(\mathrm{Fe}, \mathrm{Cu}) \mathrm{S}_{2}$, petzite $\mathrm{Ag}_{3} \mathrm{AuTe}_{2}$, utenbogardite $\mathrm{Ag}_{3} \mathrm{AuS}_{2}$, Au-bearing galena and $\mathrm{Ag}$-Sb-As sulfosalts of unusual composition are found within this association, which is estimated to be formed in the temperature range of $320-330{ }^{\circ} \mathrm{C}$ according to [38] based on arsenopyrite thermometers and fluid inclusions.

2. Late gold-sulfosalt-quartz association includes the high-grade gold (930-970\%oo) intergrown with chalcopyrite and tetrahedrite-tennantite solid-solutions, which are rather common. The specific compositions of the associated minerals are due to an increase in $\mathrm{Cu}, \mathrm{Bi}$ and $\mathrm{Sn}$ in the primary ore-forming solutions: this assemblage is characterized by cuprous phases (bornite, chalcocite, heerite, native copper, $\mathrm{Cu}-\mathrm{Zn}$ solid solutions), Bi-rich sulfosalts (aikinite $\mathrm{PbCuBiS}_{3}$, emplectite $\mathrm{CuBiS}_{2}$, witticenite $\mathrm{Cu}_{3} \mathrm{BiS}_{3}$ ) and also stannoidite $\mathrm{Cu}_{8} \mathrm{Fe}_{3} \mathrm{Sn}_{2} \mathrm{~S}_{12}$ and mawsonite $\mathrm{Cu}_{6} \mathrm{Fe}_{2} \mathrm{SnS}_{8}$. Te-free and Bi-rich tetrahedrite-tennantite series of fahlores are, as well, typomorphic minerals of this association.

3. Fluid inclusions in quartz from the gold-sulfosalt-quartz association are characterized by homogenization in the temperature range of $226-298^{\circ} \mathrm{C}$, and salinity from 0.4 to $1.2 \mathrm{wt} . \% \mathrm{NaCl}$ eq. The calculated pressures and depths of the gold-sulfosalt-quartz association formation were $2.6-8.3 \mathrm{MPa}$ and $260-530 \mathrm{~m}$, respectively.

Author Contributions: Conceptualization, methodology, writing, project administration, N.T.; sampling, general geology on the field, D.B.; microthermometric study, A.B.; preparation and description of samples, M.S. and M.P. All authors have read and agreed to the published version of the manuscript.

Funding: The studies were carried out within the framework of the state assignment of the IGM SB RAS financed by Ministry of Science and Higher Education of the Russian Federation. This research was funded by the Russian Foundation of Basic Research project No. 19-05-00316.

Data Availability Statement: The authors would like to thank N. Karmanov and M. Khlestov (IGM SB RAS) for assisting during analytical procedures.

Acknowledgments: In this section, you can acknowledge any support given which is not covered by the author contribution or funding sections. This may include administrative and technical support, or donations in kind (e.g., materials used for experiments).

Conflicts of Interest: The authors declare no conflict of interest. The funders had no role in the design of the study, collection, analyses, or interpretation of data; nor in the writing of the manuscript, or in the decision to publish the results.

\section{References}

1. Khanchuk, A.I.; Ivanov, V.V. Meso-Cenozoic geodynamic settings and gold mineralization of the Russian Far East. Russ. Geol. Geophys. 1999, 40, 1607-1617.

2. Okrugin, V.; Kokarev, S.; Okrugina, A.; ChuBarov, V.; Shuvalov, R. An unusual example of the interaction of modern hydrothermal system with Au-Ag veins (Southern Kamchatka). Miner. Mag. 1994, 58A, 669-670. [CrossRef]

3. Goryachev, N.A.; Pirajno, F. Gold deposits and gold metallogeny of Far East Russia. Ore Geol. Rev. 2014, 59, 123-151. [CrossRef]

4. Nekrasov, I.Y. Genesis of the Asachinskoe volcanogenic gold deposit in Kamchatka. Dokl. Earth Sci. 1996, 348, 94-96. (In Russian) 
5. Kim, A.U. Textural and structural features of the ores of the Asachinskoye deposit (Southern Kamchatka). In Proceedings of the Russian Conference of Students, Graduate Students and Young Scientists Dedicated to the "Year of the Planet Earth", Moscow, Russia, 6-7 April 2009; Volume 3, pp. 20-24. (In Russian).

6. Borovikov, A.; Lapukhov, A.; Borisenko, A.; Seryotkin, Y. The Asachinskoe epithermal Au-Ag deposit in Southern Kamchatka: Physicochemical conditions of formation. Russ. Geol. Geophys. 2009, 50, 693-702. [CrossRef]

7. Goryachev, N.A.; Volkov, A.V.; Sidorov, A.A.; Gamyanin, G.N.; Savva, N.E.; Okrugin, V.M. Au-Ag mineralization of volcanic belts of the North-East of Asia. Lithosphere 2010, 3, 35-50. (In Russian)

8. Andreeva, E.D.; Matsueda, H.; Okrugin, V.M.; Takahashi, R.; Ono, S. Au-Ag-Te Mineralization of the Low-Sulfidation Epithermal Aginskoe Deposit, Central Kamchatka, Russia. Resour. Geol. 2013, 63, 337-349. [CrossRef]

9. Okrugin, V.M.; Andreeva, E.D.; Yablokova, D.A.; Okrugina, A.M.; Chubarov, V.M.; Ananiev, V.V. The new data on the ores of the Aginskoye gold-telluride deposit (Central Kamchatka). In Proceedings of the Volcanism and Its Associated Processes Conference, Petropavlovsk-Kamchatsky, Russia; 2014; pp. 335-341. (In Russian).

10. Okrugin, V.M.; Andreeva, E.D.; Etschmann, B.; Pring, A.; Li, K.; Zhao, J.; Griffins, G.; Lumpkin, G.R.; Triani, G.; Brugger, J. Microporous gold: Confirmation of Au-replacing textures from nature. Am. Mineralog. 2014, 99, 1171-1175. [CrossRef]

11. Kudaeva, S.S.; Zobenko, O.A.; Yablokova, D.A.; Ulyanov, D.A. Gold of the sulfide mineral association of the Aginskoye deposit (Kamchatka). In Materials of the XIV Regional Youth Scientific Conference "The Natural Environment of Kamchatka"; Institute of Volcanology and Seismology FEB RAS: Petropavlovsk-Kamchatsky, Russia, 2015; pp. 41-50. (In Russian)

12. Corbett, G.J. Structural controls to Porphyry Cu-Au and Epithermal Au-Ag deposits in. Appl. Struct. Geol. Miner. Explor. Aust. Inst. Geosci. Bull. 2002, 36, 32-35.

13. Yablokova, D.A.; Zobenko, O.A. Pirit of the ore field Maletoyvayam (Koryakia). In Materials XII Regional Youth Scientific Conference "Research in the Field of Earth Sciences"; Institute of Volcanology and Seismology FEB RAS: Petropavlovsk-Kamchatsky, Russia, 2013; pp. 19-30. (In Russian)

14. Tolstykh, N.; Vymazalova, A.; Tuhy, M.; Shapovalova, M. Conditions of formation of Au-Se-Te minwralization in the Gaching ore occurrence (Maletoyvayam ore field), Kamchatka, Russia. Miner. Mag. 2018, 82, 649-674. [CrossRef]

15. Sidorov, E.G.; Borovikov, A.A.; Tolstykh, N.D.; Bukhanova, D.S.; Chubarov, V.M. Gold mineralization at the Maletoyvayam Deposit (Koryak Highland, Russia) and Physicochemical Conditions of its Formation. Minerals 2020, 10, 1093. [CrossRef]

16. Hedenquist, J.W.; Arribas, A.; Gonsalez-Urien, E. Exploration for epitermal gold deposits; Chapter 7. SEG Rev. Econ. Geol. 2000, $13,245-277$.

17. Hedenquist, J.W.; Arribas, R.A. Epithermal ore deposits: First-order features relevant to exploration and assessment. In Proceedings of the 14th SGA Biennial Meeting, Quebec City, QC, Canada, 20-23 August 2017; Volume 1, pp. 47-50.

18. Bolshakov, N.M.; Frolov, A.I.; Mineev, S.D.; Gazizov, R.B.; Bezrukova, L.A.; Okrugin, V.M. Geological structure of the Baranyevskoe gold ore deposit (Central Kamchatka). Domestic Geol. 2010, 4, 15-22. (In Russian)

19. Andreeva, E.D.; Konovalova, N.S. Some features of micromorphology and composition of native gold from the Baranyevskoye deposit (Central Kamchatka). In Proceedings of the Russian Conference of Students, Graduate Students and Young Scientists Dedicated to the "Year of the Planet Earth", Moscow, Russia, 6-7 April 2009; Volume 3, pp. 7-11. (In Russian).

20. Andreeva, E.D.; Kudaeva, S.S. Typomorphism of native gold of the Balkhach ore cluster. In Proceedings of the XI Youth Regional Scientific Conference, Petropavlovsk-Kamchatsky, Russia, 26 November 2013; pp. 5-18. (In Russian).

21. Lavrent'ev, Y.G.; Karmanov, N.S.; Usova, L.V. Electron-probe determination of the composition of minerals: Microanalyzer or scanning electron microscope? Russ. Geol. Geophys. 2015, 56, 1154-1161. [CrossRef]

22. Bakker, R.J. AqSo_NaCl: Computer program to calculate p-T-V-x properties in the $\mathrm{H}_{2} \mathrm{O}-\mathrm{NaCl}$ fluid system applied to fluid inclusion research and pore fluid calculation. Comput. Geosci. 2018, 115, 122-133. [CrossRef]

23. Bodnar, R.J.; Vityk, M.O. Interhretations of microthermometric data for $\mathrm{NaCl}-\mathrm{H}_{2} \mathrm{O}$ fluid inclusions. In Fluid Inclusions in Minerals: Metods and Applications; de Vivo, B., Ferozzotti, M.L., Eds.; Verginia Polytechnic Institute State University: Blacksburg, VA, USA, 1994; pp. 117-131.

24. Borisenko, A.S. Analysis of the salt composition of solutions of gas-liquid inclusions in minerals by cryometry. In The Use of Methods of Thermo Barogeochemistry in the Search and Study of Ore Deposits; Nedra: Moscow, Russia, 1982; pp. 37-47. (In Russian)

25. Roedder, E. Interpretation and Utilization of Inclusion Measurements-Compositional Data on Liquid and Gas Inclusions. In Fluid Inclusions; Ribbe, P.H., Ed.; Reviews in Mineralogy: Reston, VA, USA, 1984; Volume 12, pp. 221-250.

26. Petrenko, I.D. The Gold-Silver Deposits at Kamchtka; Publishing House of St. Petersburg VSEGEI: Petropavlovsk-Kamchatsky, Russia, 1999. (In Russian)

27. Okrugin, V.M.; Okrugina, A.M.; Andreeva, E.D.; Takahashi, R.; Matsueda, H.; Ono, S. Epithermal Mineralization of the Zolotoye Ore Field in Central Kamchatka, Russia. In Proceedings of the Society of Resource Geology, Tokyo, Japan, July 2007 ; p. 80.

28. Plotinskaya, O.Y.; Kovalenker, V.A.; Rusinov, V.L.; Zeltmann, R. Oscillatory zoning in goldfieldite of the epithermal gold deposits. Dokl. Earth Sci. 2005, 403, 799-802.

29. Plotinskaya, O.Y.; Rusinov, V.L.; Zeltmann, R. Oscillatory zoning in fahlores of epithermal gold deposits. In Crystal Chemistry and Crystal Morphology of Minerals; RMS DPI 2007-1-145-0; Petersburg Mining University Publisher: St. Petersburg, Russia, 2007; pp. 320-323. (In Russian)

30. Petrovskaya, N.V. Native Gold; Nauka: Moscow, Russia, 1973. (In Russian) 
31. Sakharova, M.S.; Demidov, V.G. On the ratio of gold and silver in the Darasun deposit. In Proceedings of A.E. Fersman Mineralogical Museum; Academy of Sciences of the USSR: Saint Petersburg, Russia, 1972; Volume 21, pp. 127-132. (In Russian)

32. Kuzhuget, R.V. Gold-Telluride Ore of The Aldan-Maadyr Ore Cluster (Western Tuva): Mineralolical and Geochemical Features of Ore and The Conditions of Their Formation. Ph.D. Thesis, Institute of Geology and Mineralogy SB RUS, Novosibirsk, Russia, 2014. (In Russian).

33. Zhuravkova, T.; Palyanova, G.; Kalinin, Y.; Goryachev, N.; Zinina, V.; Zhitova, L. Physicochemical Conditions of Formation of Gold and Silver Parageneses at the Valunistoe Deposit (Chukchi Peninsula). Russ. Geol. Geophys. 2019, 60, 1247-1256. [CrossRef]

34. Prince, A.; Velikanova, T.; Turchanin, M.A. Silver-Gold-Copper. In Landolt-Boernstein: Numerical Data and Functional Relationships in Science and Technology (New Series); Group IV: Physical Chemistry; "Ternary Alloy Systems: Phase Diagrams, Crystallographic and Thermodynamic Data"; Martiensen, W., Effenberg, G., Ilyenko, S., Eds.; Springer: Berlin/Heidelberg, Germany, 2006; Volume 11B, pp. 10-41.

35. Chudnenko, K.V.; Palyanova, G.A. Thermodynamic modeling of native formation of Au-Ag-Cu-Hg solid solutions. Appl. Geochem. 2016, 66, 88-100. [CrossRef]

36. Okrugin, V.M.; Yablokova, D.A.; Andreeva, E.D.; Shishkanova, K.O.; Chubarov, V.M.; Filosofova, T.M.; Moskaleva, S.V.; Chernev, I.I.; Chubarov, M.V. New data on pyrite of modern and paleohydrothermal systems of Kamchatka. In Materials of the Annual Conference Dedicated to the Volcanologist's Day "Volcanism and Related Processes"; IVS FEB RAS Publisher: Petropavlovsk-Kamchatsky, Russia, 2014; pp. 324-329. (In Russian)

37. Tang, Q.; Di, P.; Yu, M.; Bao, J.; Zhao, Y.; Liu, D.; Wang, Y. Mineralogy and geochemistry of pyrite and arsenopyrite from the zaozigou gold deposit in West Qinling Orogenic Beld, Central China: Implications for ore genesis. Resour. Geol. 2019, 69, 314-332. [CrossRef]

38. Zachariáš, J.; Frýda, J.; Paterová, B.; Mihaljevič, M. Arsenopyrite and As-bearing pyrite from the Roudný deposit, Bohemian Massif. Miner. Mag. 2004, 68, 31-46. [CrossRef]

39. Cepedal, A.; Fuertes-Fuente, M.; Martin-Izard, A. Gold-bearing As-ric h pyrite and arsenopyrite from the el valle gold deposit, Asturias, Northwestern Spain. Can. Miner. 2008, 46, 233-247. [CrossRef]

40. Lyubimtseva, N.G.; Bortnikov, N.S.; Borisovskiy, S.E. Oscillatory zoning of tennantite- (fe) gold ore of the deposit Darasun (Eastern Transbaikalia, Russia). Geol. Depos. 2020, 62, 195-223. (In Russian)

41. Foord, E.E.; Shawe, D.R. The Pb-Bi-Ag-Cu-(Hg) chemistry of galena and some associated sulfosalts: A review and some new data from Colorado, California and Pennsylvania. Can. Miner. 1989, 27, 363-382.

42. Vakh, A.S.; Avchenko, O.V.; Gvozdev, V.I.; Goryachev, N.A.; Karabtsov, A.A.; Vakh, E.A. Minerals of the Pb-As-Sb-S and $\mathrm{Cu}-\mathrm{Pb}-\mathrm{As}-\mathrm{Sb}-\mathrm{S}$ systems in the ores of the Berezitovoe gold-polymetal deposit (Upper Amur region, Russia). Geology Depos. 2019, 61, 64-84. (In Russian) [CrossRef]

43. Sack, R.O.; Fredericks, R.; Hardy, L.S.; Ebel, D.S. Origin of high-Ag fahlores from the Galena Mine, Wallace, ID, USA. Am. Miner. 2005, 90, 1000-1007. [CrossRef]

44. Staude, S.; Mordhorst, T.; Neumann, R.; Prebeck, W.; Märkl, G. Compositional variation of the tennantite-tetrahedrite solidsolution series in the Schwarzwald ore district (SW Germany): The role of mineralization processes and fluid source. Miner. Mag. 2010, 74, 309-339. [CrossRef]

45. Godovikov, A.A. Bismuth Sulfosalts; Nauka Publishing House: Moscow, Russia, 1972. (In Russian)

46. Makovicky, E.; Karup-Møller, S. Exploratory Studies of Substitutions in the Tetrahedrite/Tennantite-Goldfieldite Solid Solution. Can. Miner. 2017, 55, 233-244. [CrossRef]

47. Kovalenker, V.A. The first discovery of mawsonite and other CuFe-Sn sulfides in gold-sulfide-quartz ores. Int. Geol. Rev. 1981, 23, 105-114. [CrossRef]

48. Kovalenker, V.A.; Plotinskaya, O.Y.; Prokofev, V.Y.; Gertman, Y.L.; Koneev, R.I.; Pomortsev, V.V. Mineralogy, geochemistry, and genesis of gold-sulfide-selenide-telluride ores from the Kairagach deposit (Uzbekistan). Geol. Ore Depos. 2003, 45, 171-200.

49. Takahashi, R.; Matsueda, H.; Okrugin, V. Epithermal gold and silver mineralization at the Rodnikovoe deposit related to the hydrothermal activity in the Mutnovsko-Asachinskaya geothermal area, Southern Kamchatka, Russia. In Proceedings of the International Symposium on Gold and Hydrothermal Systems, Fukuoka, Japan, 4 November 2001; pp. 51-57.

50. Takahashi, R.; Matsueda, H.; Okrugin, V.M.; Ono, S. Epithermal Gold-Silver Mineralization of the Asachinskoe Deposit in South Kamchatka, Russia. Resour. Geol. 2007, 57, 354-373. [CrossRef]

51. Mehrabi, B.; Siani, M.G. Intermediate sulfidation epithermal Pb-Zn-Cu ( $\pm \mathrm{Ag}-\mathrm{Au})$ mineralization at Cheshmeh Hafez deposit, Semnan Province. J. Geol. Soc. India 2012, 80, 563-578. [CrossRef]

52. Hedenquist, J.; Henley, R.W. Hydrothermal eruptions in the Waiotapu geothermal system, New Zealand; their origin, associated breccias, and relation to precious metal mineralization. Econ. Geol. 1985, 80, 1640-1668. [CrossRef]

53. Corbett, G. Epithermal Au-Ag Deposit Types-Implications for Exploration. 2005. Available online: https://www.researchgate. net/publication/237489786 (accessed on 30 August 2014).

54. Prokof'ev, V.Y.; Ali, A.A.; Volkov, A.V.; Savva, N.E.; Kolova, E.E.; Sidorov, A.A. Geochemical peculiarities of ore forming fluidof the Juliette Au-Ag epithermal deposit (Northeastern Russia). Dokl. Earth Sci. 2015, 460, 87-91. [CrossRef]

55. Mancano, D.; Campbell, A. Microthermometry of enargite-hosted fluid inclusions from the Lepanto, Philippines, high-sulfidation CuAu deposit. Geochim. Cosmochim. Acta 1995, 59, 3909-3916. [CrossRef] 
56. Alekseev, V.Y.; Savva, N.E.; Kolova, E.E. The Agan Lapukhov, A.S.; Borovikov, A.A.; Guzman, B.V.; Miroshnichenko, L.V.; Rasvorotneva, L.I. Hieratite in hydrothermally altered volcanic rocks of Danchenkovskoye deposit (the Urup Island). Zap. RMO 2012, 141, 52-59. (In Russian)

57. Volkov, A.V.; Savva, N.E.; Sidorov, A.A.; Kolova, E.E.; Chizhova, I.A.; Alekseev, V.Y. The Agan epithermal gold-silver deposit and prospects for the discovery of high-sulfidation mineralization in Northeast Russia. Geol. Ore Depos. 2015, 57, 21-41. [CrossRef]

58. Sahlström, F.; Arribas, A.; Dirks, P.; Corral, I.; Chang, Z. Mineralogical Distribution of Germanium, Gallium and Indium at the Mt Carlton High-Sulfidation Epithermal Deposit, NE Australia, and Comparison with Similar Deposits Worldwide. Minerals 2017, 7, 213. [CrossRef]

59. Volkov, A.V.; Chizhova, I.A.; Alekseev, V.Y.; Sidorov, A.A. Variations of the Ag/Au index in epithermal deposits. Dokl. Earth Sci. 2013, 452, 911-914. [CrossRef] 